\title{
The systematic position of Dryopteris blanfordii subsp. nigrosquamosa (Ching) Fraser-Jenkins within the genus Dryopteris Adans.
}

\author{
Anastasiya A. Krinitsina', Maxim S. Belenikin², \\ Olga A. Churikova', Sergey V. Kuptsov³, Maxim I. Antipin ${ }^{3}$, \\ Maria D. Logacheva ${ }^{4}$, Anna S. Speranskaya'
}

I Department of High Plants, Biological Faculty, Lomonosov Moscow State University, Leninskie gory, 1, 12, Moscow, Russia, 1192342 Department of Molecular and Biological Physics, Moscow Institute of Physics and Technology, Dolgoprudny, Moscow Region, Russia, 1417003 Botanical Garden, Lomonosov Moscow State University, Leninskie gory, Moscow, Russia, 1198994 Department of Evolutional Biochemistry, A.N. Belozersky Institute of PhysicoChemical Biology, Lomonosov Moscow State University, Leninskie gory, 1, 40, Moscow, Russia, 119992

Corresponding author: Anastasiya A. Krinitsina (ankrina@gmail.com)

Academic editor: T. Almeida | Received 28 June 2017 | Accepted 6 October 2017 | Published 15 November 2017

Citation: Krinitsina AA, Belenikin MS, Churikova OA, Kuptsov SV, Antipin MI, Logacheva MD, Speranskaya AS (2017) The systematic position of Dryopteris blanfordii subsp. nigrosquamosa (Ching) Fraser-Jenkins within the genus Dryopteris Adans. PhytoKeys 90: 89-112. https://doi.org/10.3897/phytokeys.90.14745

\begin{abstract}
Dryopteris blanfordii (C.Hope) C.Chr. is a member of the Dryopteridaceae, growing in high altitude Picea or Abies forests (2900-3500 m) in China and India. Phylogenetic relationships between D. blanfordii subsp. nigrosquamosa and closely related species of Dryopteris were investigated using a combined analysis of multiple molecular data sets (the protein-coding region of $r b c L$ and matK genes and intergenic spacers $p s b A$-trnH, trnP-pet $G$, rps4-trnS, trnL-trnF and $r b c L$-accD). An assumption about the position of D. blanfordii subsp. nigrosquamosa within Dryopteris was made by using the Maximum Likelihood and Bayesian Inference approach and chloroplast marker sequences of Dryopteris species from GenBank. The results demonstrated that Asian taxa $D$. blanfordii subsp. nigrosquamosa and $D$. laeta as well as two American species D. arguta and D. marginalis belong to the same clade, all four of them being part of Dryopteris section Dryopteris.
\end{abstract}

\section{Keywords}

chloroplast genome markers, Dryopteridaceae, Dryopteris blanfordii subsp. nigrosquamosa, phylogeny 


\section{Introduction}

Dryopteris is a large fern genus of some 225-230 species belonging to the Dryopteridaceae (Fraser-Jenkins 1986, Kramer 1990, Zhang et al. 2012). Dryopteris blanfordii (C.Hope) C.Chr. grows in Picea or Abies forests at 2900-3500 m in China (Gansu, Sichuan, Xizang, Yunnan), Afghanistan, India, Kashmir, Nepal and Pakistan (Zhang et al. 2013). It is divided into two subspecies, subsp. nigrosquamosa (Ching) FraserJenkins and subsp. blanfordii. Dryopteris blanfordii subsp. nigrosquamosa is abundant in India (the Kashmir valley) (Mir et al. 2014, Mir et al. 2015), China (Gansu, Sichuan, Xizang, Yunnan) and Nepal (Zhang et al. 2013).

The current taxonomy and infrageneric position of $D$. blanfordii are still unresolved. More than a quarter of a century ago, Fraser-Jenkins (1986) divided Dryopteris into several sections based on the comparison of macro- and micro-morphological traits. He established Sect. Remotae with a single species (D. blanfordii) in the Indian subcontinent and with two European species (Dryopteris remota Hayek. and D. corleyi Fraser-Jenk.). Fraser-Jenkins suggested that all species from Sect. Remotae are allopolyploids resulting from hybridization between species from different sections (such as sect. Fibrillosae, sect. Lophodium or sect. Marginatae) (Fraser-Jenkins 1986).

In a recent study, a phylogeny including 100 species of Dryopteris was reconstructed and 13 phylogenetic clades (or major evolutionary lineages) were identified using DNA sequences of four plastid loci $(r b c L$ gene, $r p s 4$-trnS spacer, $\operatorname{trn} L$ intron and $\operatorname{trnL}$ $F$ spacer) (Zhang et al. 2012). Two of the three species, previously placed in Sect. Remotae, were identified in this study as members of other clades, namely clade Aemulae (D. corleyi) and clade Lophodium (D. remota) (Zhang et al. 2012). Similar results were demonstrated by Sessa et al. (2012a, 2012b), where seven plastid loci (rbcL, psbA$\operatorname{trn} H, \operatorname{trn} P-p e t G, \operatorname{rps} 4-\operatorname{trn} S, \operatorname{trn} L-F, \operatorname{trn} G-\operatorname{trn} R$ and $r b c L-a c c D)$ and a single nuclear marker $p g i C$ were used to analyse 97 Dryopteris species. The data of these phylogenies, although valuable, were far from complete and the taxonomic position of $D$. blanfordii subsp. nigrosquamosa remained unclear.

\section{Material and methods}

\section{Plant material}

To examine the morphology of $D$. blanfordii subsp. nigrosquamosa, adult plants from the Botanical Garden of Moscow State University were used. The parent plant of Dryopteris blanfordii (C.Hope) C.Chr. subsp. nigrosquamosa (Ching) Fraser-Jenk was collected in 2003 in Uttar Pradesh State, India, at 3000 m. Spores of the specimen were germinated under artificial conditions in the greenhouse complex of MSU Botanical Garden. Subsequently, developed sporophytes were transplanted to the outdoor section of the Botanical Garden. The adult specimens were used for DNA sampling. The voucher specimen was deposited at Herbarium MW. Reference morphological char- 
acters for $D$. blanfordii subsp. nigrosquamosa were scored from a type specimen (PE 00133945, locality: Tibet) and from descriptions of Dryopteris blanfordii (C.Hope) C.Chr. subsp. nigrosquamosa (Zhang et al. 2013, Mir et al. 2014, Mir et al. 2015).

\section{Chloroplast markers sequencing and assembling}

The chloroplast marker sequences of $D$. blanfordii subsp. nigrosquamosa were obtained during a large project on Polypodiales chloroplast genome sequencing. Sequencing data were generated using Illumina MiSeq high-throughput sequencing platform. For sample preparation, adult living plants were taken from the collection of the MSU Botanical Garden. The cpDNA fraction was extracted from $2.6 \mathrm{~g}$ (fresh weight) of fronds using a slightly modified cpDNA extraction protocol (Shi et al. 2012, Vieira et al. 2014). The purification of DNA was carried out using a protocol designed by the authors (Krinitsina et al. 2015). TruSeq protocol (NEBNext ${ }^{\circ}$ DNA Library Prep Master Mix Set for Illumina, E6040, NEB reagents) was used for preparing the libraries. Pare end $(\mathrm{PE})$ sequences $(2 \times 300 \mathrm{bp})$ with a double number of each library reads about $1.2-1.97 \mathrm{M}$ were made. After quality trimming by Trimmomatic (Bolger et al. 2014), reads were filtered using 13 complete and five partial fern chloroplast genome sequences from RefSeq database and Bowtie2 (Langmead and Salzberg 2012). Then two sets of contigs were produced for both filtered and unfiltered sets of reads using Velvet Assembler (Zerbino and Birney 2008) and MIRA4 (Chevreux et al. 2004). Assembled contigs and scaffolds were used for assembling the complete chloroplast genome (the data are not presented in this paper) and for extracting target chloroplast markers, namely $r b c L$, matK genes and intergenic spacers $p s b A-t r n H$, trnP-pet $G$, rps4trnS, trnL-trnF and $r b c L-a c c D$.

\section{Dryopteris blanfordii subsp. nigrosquamosa phylogenetic analysis}

To determine the phylogenetic position of $D$. blanfordii subsp. nigrosquamosa, a phylogenetic analysis using sequences published in GenBank was performed. The GenBank accession numbers of sequences of Dryopteris species included in this study are listed in Appendix 1. Sequence alignment was conducted using Muscle algorithm and MEGA6.0 software package (www.megasoftware.net, Tamura et al. 2013). Phylogenetic analyses were performed using the Maximum Likelihood (ML) method at MEGA 6.0 (Tamura et al. 2013) and Bayesian Inference (BI) in BEAST (Bouckaert et al. 2014). A combined matrix including $m a t K$ and $r b c L$ gene and five intergenic spacers (psbA-trnH, rps4-trnS, trnL-trnF trnP-pet G and $r b c L$-accD) of 84 Dryopteris species (including $D$. blanfordii subsp. nigrosquamosa) was analysed.

A bootstrapping of 1000 replicates for ML analysis was processed to estimate the confidence probabilities on each branch of the phylogenetic trees constructed. The initial tree (ML) for heuristic search was obtained by applying the Neighbour-Joining 
method to a matrix of pairwise distances estimated using the Maximum Composite Likelihood approach (Lindsay 1988, Tamura et al. 2013). All positions containing gaps and missing data were eliminated.

Bayesian analyses were run for 20,000,000 generations with four MCMC chains in two independent runs. The first 2,000,000 samples from each run were discarded as burn-in. Convergence was assessed by comparing the standard deviation of split frequencies between different runs (MCMC Trace Analysis Tool (Tracer) version v1.6.0 (Rambaut et al. 2014). For ML and BI analyses, optimal models of molecular evolution for combined matrices were identified using jModelTest2 (Darriba et al. 2012) through Bayesian Information Criterion (BIC).

\section{Results}

Dryopteris blanfordii subsp. nigrosquamosa phylogenetic position

Seven marker regions of the assembled cp genome were used for determining phylogenetic relationships between D. blanfordii subsp. nigrosquamosa and other Dryopteris species, i.e. protein-coding regions of $r b c L$ and matK genes and intergenic spacers $p s b A-\operatorname{trn} H, \operatorname{trn} P-p e t G, r p s 4-\operatorname{trn} S, \operatorname{trnL}-\operatorname{trn} F$ and $r b c L-a c c D$. These markers were assembled into a single data matrix consisting of 3734 total bases. The optimal model of molecular evolution for combined matrices was TPM1uf $+\mathrm{G}+\mathrm{I}$ with $\mathrm{BIC}=36592.7258$. The phylogenetic tree is shown in Fig. 2. The analysis demonstrated close relationships between $D$. blanfordii subsp. nigrosquamosa, D. laeta, D. marginalis and D. arguta. The clades containing $D$. blanfordii subsp. nigrosquamosa were well-supported ( $\geq 80 \%$ bootstrap support). Dryopteris blanfordii subsp. nigrosquamosa is close to D. laeta (bootstrap $=100 / \mathrm{PP}=100 \%), D$. arguta (bootstrap $=87 / \mathrm{PP}=99.6 \%$ ) and $D$. marginalis (bootstrap $=96 / \mathrm{PP}=100 \%)$. The results of Bayesian Inference analysis based on the combined matrix were highly congruent with the strict consensus tree from ML analysis. The clade that included D. arguta, D. marginalis, D. laeta and D. blanfordii subsp. nigrosquamosa in the combined matrix of seven markers had the posterior probability (PP) value of $100 \%$.

Morphological characters of $D$. blanfordii subsp. nigrosquamosa and closely related species.

The adult specimens of $D$. blanfordii analysed in the present work have $55-57 \times 30-35 \mathrm{~cm}$ fronds. The frond dissection is 2-pinnate with symmetrical pinnae and pinnules (Fig. 1A). The rachises and petioles are fibrillose and have dense basal scales. The scales on the petioles are dark-brown basally and light-brown at the apex (Fig. $1 \mathrm{~B}$ and C). The costa and rachises are slightly grooved adaxially (Fig. 1D) and rounded abaxially (Fig. 1E). 


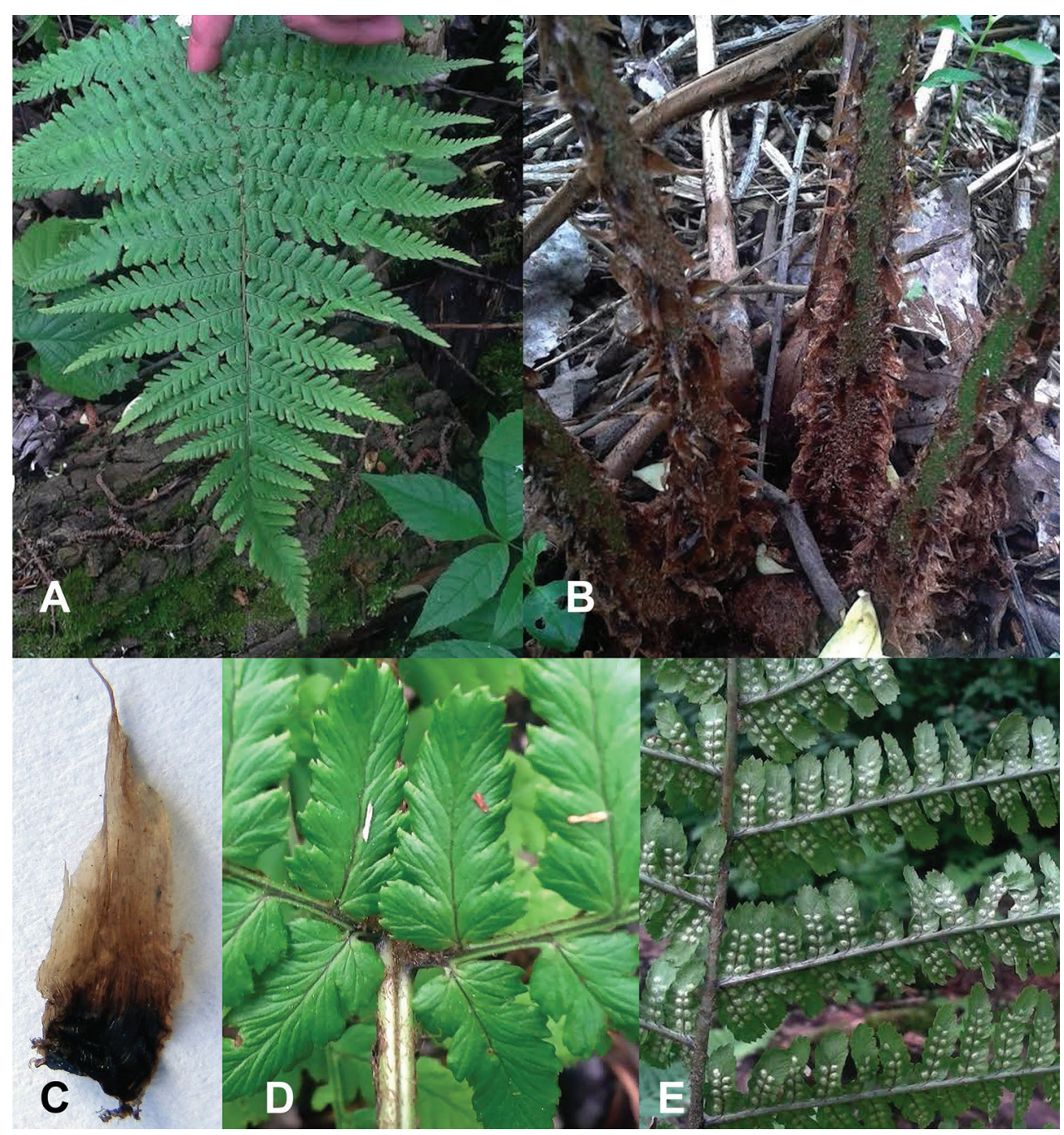

Figure I. Dryopteris blanfordii subsp. nigrosquamosa morphology. A Frond of mature plants B Petiole covered with scales $\mathbf{C}$ Petiole scale $\mathbf{D}$ Adaxial surface of rachis and costa $\mathbf{E}$ Abaxial surface of rachis and costa.

Two closely related species, namely $D$. arguta and $D$. marginalis, are native to North America. Dryopteris marginalis is evergreen and has tawny or cinnamon-coloured scales, lanceolate and coriaceous laminae, with sori mostly at margins of ultimate pinnules' segments (Table 1). Dryopteris arguta is winter green, having grassygreen to yellow-green, ovate-lanceolate, herbaceous, glandular laminae; the basal basiscopic pinnule and basal acroscopic pinnule are \pm equal; its pinnule margins are serrate with spreading, spinelike teeth; the sori are medial. Both North American species have longer stipes (1/4-1/3 length of leaf), 1-pinnate-pinnatifid to 2-pinnate-pinnatifid fronds (Montgomery and Wagner 1993). 
Table I. Morphological characters of four species of Dryopteris: D. blanfordii subsp. nigrosquamosa, D. laeta, D. marginalis and D. arguta according to Montgomery and Wagner (1993), Mir et al. (2014), Mir et al. (2015).

\begin{tabular}{|c|c|c|c|c|}
\hline Species & $\begin{array}{l}\text { D. blanfordii subsp. } \\
\text { nigrosquamosa }\end{array}$ & D. laeta & D. marginalis & D. arguta \\
\hline $\begin{array}{l}\text { Natural } \\
\text { range }\end{array}$ & $\begin{array}{c}\text { Southeast Tibet, } \\
\text { Western China, Nepal, } \\
\text { India (Kashmir) }\end{array}$ & $\begin{array}{c}\text { North China, Eastern } \\
\text { Siberia, North Korea } \\
\text { and North Japan }\end{array}$ & Eastern North America & $\begin{array}{c}\text { From British } \\
\text { Columbia to Baja } \\
\text { California } \\
\end{array}$ \\
\hline Seasonality & semi-evergreen & \begin{tabular}{|l|} 
deciduous \\
\end{tabular} & evergreen & winter green \\
\hline Rhizomes & erect & creeping & ascending to erect & short-creeping \\
\hline Scale colour & $\begin{array}{l}\text { light brown with } \\
\text { black veins }\end{array}$ & pale brown & tawny to cinnamon & light brown \\
\hline $\begin{array}{l}\text { Lamina } \\
\text { length }(\mathrm{cm})\end{array}$ & $40-75$ & $25-50$ & $25-50(75)$ & $25-90$ \\
\hline $\begin{array}{l}\text { Lamina } \\
\text { division }\end{array}$ & $\begin{array}{c}\text { 2-pinnate to } \\
\text { 3-pinnate-pinnatifid }\end{array}$ & 3-pinnate-pinnatifid & $\begin{array}{l}\text { 1-pinnate-pinnatifid to } \\
\text { 2-pinnate-pinnatifid }\end{array}$ & $\begin{array}{l}\text { 2-pinnate- } \\
\text { pinnatifid }\end{array}$ \\
\hline $\begin{array}{l}\text { Lamina } \\
\text { colour and } \\
\text { texture }\end{array}$ & $\begin{array}{l}\text { glaucous green, } \\
\text { coriaceous }\end{array}$ & $\begin{array}{l}\text { green, herbaceous to } \\
\text { thinly papyraceous }\end{array}$ & green, coriaceous & $\begin{array}{l}\text { green to yellow- } \\
\text { green, herbaceous, } \\
\text { glandular }\end{array}$ \\
\hline $\begin{array}{l}\text { Lamina } \\
\text { shape }\end{array}$ & $\begin{array}{c}\text { lanceolate to oblong- } \\
\text { lanceolate }\end{array}$ & $\begin{array}{c}\text { ovate-oblong or } \\
\text { deltoid-ovate }\end{array}$ & ovate-lanceolate & ovate-lanceolate \\
\hline Stipe length & $\begin{array}{c}1 / 5-1 / 4 \text { of rachis } \\
\text { length }\end{array}$ & $\begin{array}{c}1 / 3 \text { to } 1 / 2 \text { of rachis } \\
\text { length }\end{array}$ & $\begin{array}{c}1 / 4 \text { to } 1 / 3 \text { of rachis } \\
\text { length }\end{array}$ & $\begin{array}{c}1 / 4 \text { to } 1 / 3 \text { of rachis } \\
\text { length }\end{array}$ \\
\hline $\begin{array}{l}\text { Sori } \\
\text { arrangement }\end{array}$ & $\begin{array}{l}\text { in } 1 \text { row at each } \\
\text { side of midvein, } \\
\text { inframedial } \\
\end{array}$ & $\begin{array}{l}\text { in } 2 \text { rows at each } \\
\text { side of midvein }\end{array}$ & $\begin{array}{l}\text { in } 1 \text { row at each side of } \\
\text { midvein, intramarginal } \\
\text { at margins of segments }\end{array}$ & $\begin{array}{l}\text { in } 1 \text { row at each } \\
\text { side of midvein, } \\
\text { medial }\end{array}$ \\
\hline
\end{tabular}

Dryopteris laeta is characterised by a long stipe (length roughly equal to blade length) with very few lanceolate scales; deciduous, ovate-oblong or deltoid-ovate, 3 -pinnate-pinnatifid, $25-50 \times 15-40 \mathrm{~cm}$, herbaceous to thinly papyraceous laminae; pinnules with toothed margins ending in an acute apex; sori in 1 or 2 rows on each side of pinnule costa; indusia orbicular-reniform, membranaceous, margin eroded (Zhang et al. 2013). The main morphological characters of these four species of Dryopteris are presented in the table below.

\section{Discussion}

The data obtained in this study allowed us to suggest a more accurate view of the taxonomic position of $D$. blanfordii subsp. nigrosquamosa. Our results demonstrated that $D$. laeta and D. blanfordii subsp. nigrosquamosa belong to the same clade as D. arguta and $D$. marginalis. According to the classification system of the genus Dryopteris by FraserJenkins (1986), D. arguta and D. marginalis belong to sect. Pallidae, while D. blanfordii belongs to sect. Remotae. More recent classifications divide the genus Dryopteris into either five (Sessa et al. 2012b) or 13 different clades (Zhang et al. 2012). We have concluded that $D$. blanfordii subsp. nigrosquamosa together with $D$. laeta, D. arguta and D. marginalis belong to the Dryopteris clade following Zhang et al. (2012) or clade I according 

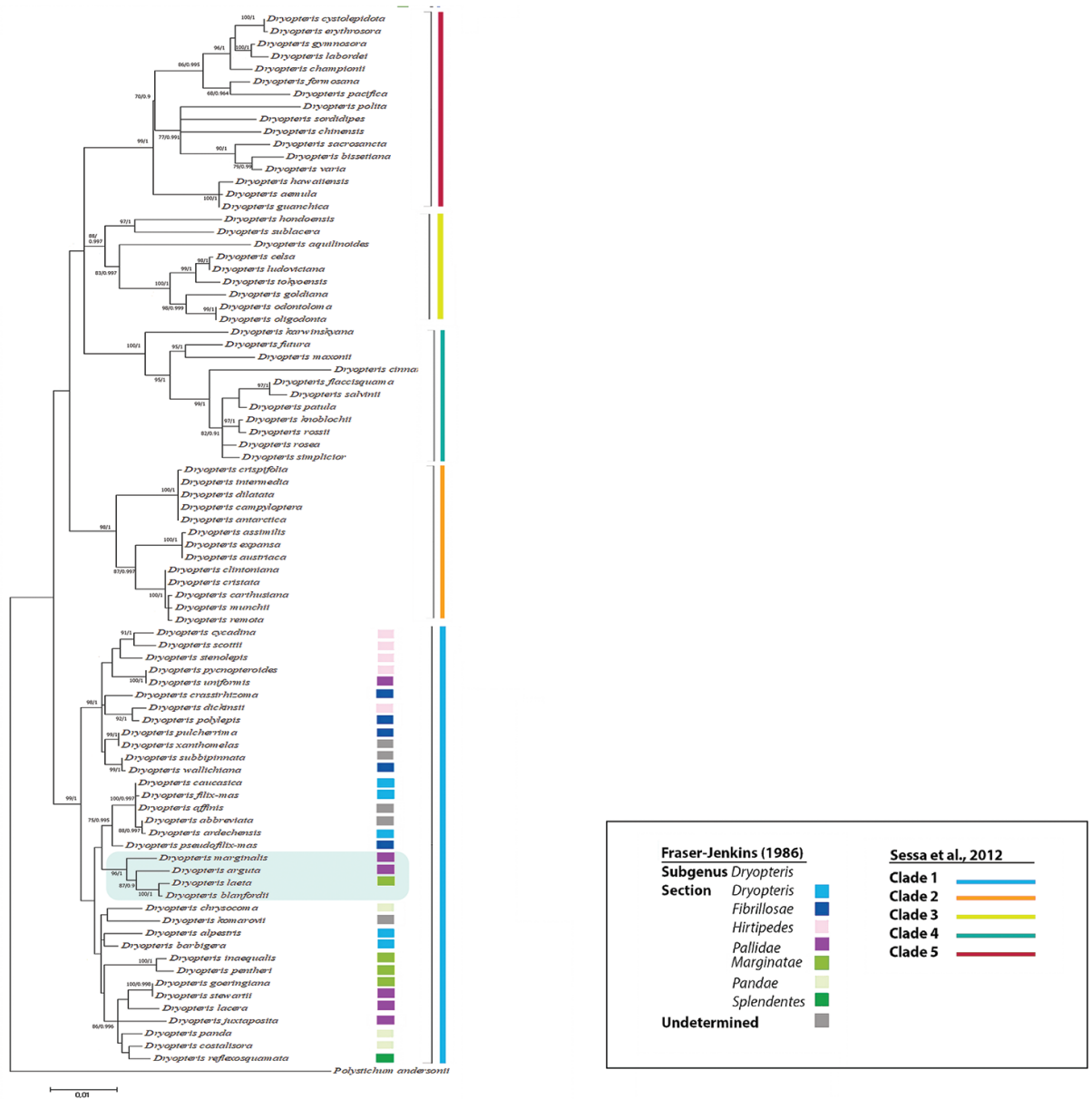

Figure 2. Topology from $M L$ and $B I$ analyses of Dryopteris species using six marker regions consisting of 3734 total bases ( $p s b A-t r n H, r b c L-a c c D, r b c L, \operatorname{trnL}-\operatorname{trn} F, \operatorname{trn} P$-petG, rps4-trnS and matK). The tree is drawn to scale, with branch lengths measured in the number of substitutions per site. Bootstrap values are specified at the branch nodes (cut off $>50 \%$ ) / Bayesian PP. Dryopteris blanfordii subsp. nigrosquamosa and closely related species are marked with a blue rectangle.

to Sessa et al. (2012b). Dryopteris arguta and D. marginalis are closely related American species (from western and eastern parts of North America respectively) and the D. laeta specimen is from a population located in Iwaizumi (Iwate prefecture, Japan). According to some recent studies (Widén et al. 2015), Dryopteris goeringiana (Kunze) Koidz. is a synonym of $D$. laeta, growing in Japan. Our analyses rather showed that $D$. goeringiana proves to be related to Dryopteris stewartii Fraser-Jenk., Dryopteris lacera (Thunb.) Kunze and Dryopteris sieboldii (Van Houtte ex Mett.) Kuntze (Fig. 2), which agrees with the data of other authors (Sessa et al. 2012b). At the same time, D. laeta from Japan does not belong to this group of Dryopteris species (Ebihara 2011, Zhang et al. 2012) (Fig. 2). The relationship between species from Europe, Central America and Asia may indicate that a 
long-distance dispersal event occurred. Unfortunately, it is impossible to indicate where the ancestor of these species might have originated.

Dryopteris arguta, D. marginalis, D. laeta and D. blanfordii subsp. nigrosquamosa differ from each other in many morphological characters (Table 1), but share the structure of segments of second and third orders. The second order segments (pinnules) are short- or very short-stalked $(1-5(10) \mathrm{mm})$. The distal part of the pinnule is attenuated and elongated, with its distal third alate and lacking sori. The basal basiscopic to acroscopic pinnulae length ratio is $1-1.5$. The third order segments, when present, are isomorphic. Pinnules are serrate, ending with more or less prominent spiny teeth.

\section{Conclusion}

The relationship of $D$. blanfordii subsp. nigrosquamosa within the Dryopteris genus was defined using phylogenetic analyses based on chloroplast markers. Our results demonstrate that Asian species D. blanfordii subsp. nigrosquamosa and D. laeta belong to the same clade as two North American species D. arguta and D. marginalis, with all four species being part of the Dryopteris section Dryopteris.

\section{Acknowledgements}

This investigation (except sequencing works) was supported by the Russian Foundation for Basic Research, grant no. 14-04-01852a. The sequencing works were supported by Russian Science Foundation grant no. 14-50-00029.

\section{References}

Bolger AM, Lohse M, Usadel B (2014) Trimmomatic: a flexible trimmer for Illumina sequence data. Bioinformatics 30(15): 2114-2120. https://doi.org/10.1093/bioinformatics/btu170

Bouckaert R, Heled J, Kuhnert D, Vaughan T, Wu CH, Xie D, Suchard MA, Rambaut A, Drummond AJ (2014) BEAST 2: A Software Platform for Bayesian Evolutionary Analysis. PLoS Computational Biology 10(4): e1003537. https://doi.org/10.1371/journal.pcbi.1003537

Chevreux B, Pfisterer T, Drescher B, Driesel AJ, Müller WE, Wetter T, Suhai S (2004) Using the miraEST Assembler for Reliable and Automated mRNA Transcript Assembly and SNP detection in sequenced ESTs. Genome research 14: 1147-1159. https://doi.org/10.1101/gr.1917404

Darriba D, Taboada GL, Doallo R, Posada D (2012) jModelTest 2: more models, new heuristics and parallel computing. Nature Methods 9(8): 772. https://doi.org/10.1038/nmeth.2109

Ebihara A (2011) RbcL phylogeny of Japanese pteridophyte flora and implications on infrafamilial systematics. Bulletin of the National Museum of Nature and Science, Ser. B, botany 37(2): 63-74. 
Fraser-Jenkins R (1986) A classification of the genus Dryopteris (Pteridophyta: Dryopteridaceae). Bulletin of British Museum (Natural History), Botany series 14(3): 183-218.

Kramer KU (1990) Dryopteridaceae. In: Kramer KU, Green PS (Eds) The Families and Genera of Vascular Plants. Vol. 1. Pteridophytes and Gymnosperms. Springer, Berlin, 101-144. https://doi.org/10.1007/978-3-662-02604-5_23

Krinitsina AA, Sizova TV, Zaika MA, Speranskaya AS, Sukhorukov AP (2015) A rapid and cost-effective method for DNA extraction from archival herbarium specimens. Biochemistry (Moscow) 80(11): 1478-1484. https://doi.org/10.1134/S0006297915110097

Langmead B, Salzberg S (2012) Fast gapped-read alignment with Bowtie 2. Nature Methods 9: 357-359. https://doi.org/10.1038/nmeth.1923

Lindsay BG (1988) Composite Likelihood Methods. Contemporary Mathematics 80: 221-239. https://doi.org/10.1090/conm/080/999014

Mir SA, Mishra AK, Reshi ZA, Sharma MP (2014) Four newly recorded species of Dryopteridaceae from Kashmir valley, India. Biodiversitas 15(1): 6-11. https://doi.org/10.13057/ biodiv/d150102

Mir SA, Mishra AK, Pala SA, Reshi ZA, Sharma MP (2015) Ferns and fern allies of District Shopian, Kashmir Valley, India. Biodiversitas 16(1): 27-43. https://doi.org/10.13057/ biodiv/d160105

Montgomery JD, Wagner WH (1993) Dryopteris. In: Flora of North America Editorial Committee (Eds) Flora of North America North of Mexico, vol. 2. Oxford University Press, New York, 280-288.

Rambaut A, Suchard MA, Xie D, Drummond AJ (2014) Tracer v1.6. Available from http:// beast.bio.ed.ac.uk/Tracer

Sessa EB, Zimmer EA, Givnish TJ (2012a) Reticulate evolution on a global scale: a nuclear phylogeny for New World Dryopteris (Dryopteridaceae). Molecular Phylogenetics and Evolution 64(3): 563-581. https://doi.org/10.1016/j.ympev.2012.05.009

Sessa EB, Zimmer EA, Givnish TJ (2012b) Phylogeny, divergence times, and historical biogeography of New World Dryopteris (Dryopteridaceae). American Journal of Botany 99(4): 730-750. https://doi.org/10.3732/ajb.1100294

Sessa EB, Zimmer EA, Givnish TJ (2012c) Unraveling reticulate evolution in North American Dryopteris (Dryopteridaceae). BMC Evolutionary Biology 12: 104. https://doi. org/10.1186/1471-2148-12-104

Shi C, Hu N, Huang H, Gao J, Zhao YJ, Gao LZ (2012) An improved chloroplast DNA extraction procedure for whole plastid genome sequencing. PLoS One 7(2): e31468. https:// doi.org/10.1371/journal.pone.0031468

Tamura K, Stecher G, Peterson D, Filipski A, Kumar S (2013) MEGA6: Molecular Evolutionary Genetics Analysis version 6.0. Molecular Biology and Evolution 30: 2725-2729. https://doi.org/10.1093/molbev/mst197

Vieira LN, Faoro H, Fraga HPF., Rogalski M, de Souza EM, Pedrosa FO, Nodari RO, Guerra M (2014) An improved protocol for intact chloroplasts and cpDNA isolation in conifers. PLoS One 9(1): e84792. https://doi.org/10.1371/journal.pone.0084792

Widén C-J, Fraser-Jenkins CR, Roux JP (2015) A survey of phenolic compounds in Dryopteris and related fern genera. Part IV. Phloroglucinol derivatives and morphology in the sec- 
tion Marginate (Pteridophyta, Dryipteridaceae). Annales Botanici Fennici 52(1-2): 53-58. http://dx.doi.org/10.5735/085.052.0209

Zerbino DR, Birney E (2008) Velvet: algorithms for de novo short read assembly using de Bruijn graphs. Genome Research 18(5): 821-829. https://doi.org/10.1101/ gr.074492.107

Zhang LB, Zhang L, Dong SY, Sessa EB, Gao XF, Ebihara A (2012) Molecular circumscription and major evolutionary lineages of the fern genus Dryopteris (Dryopteridaceae). BMC Evolutionary Biology 12: 180. https://doi.org/10.1186/1471-2148-12-180

Zhang LB, Wu SG, Xiang JY, Xing FW, He H, Wang FG, Lu SG, Dong SY, Barrington DS, Iwatsuki K, Christenhusz MJM, Mickel JT, Kato M, Gilbert MG (2013) Dryopteridaceae. In: Wu Z-Y, Raven PH, Hong D-Y (Eds) Flora of China, vols. 2-3. Science Press, Beijing; Missouri Botanical Garden Press, St. Louis, 541-724. 


\begin{tabular}{|c|c|c|c|c|c|c|c|c|c|c|}
\hline 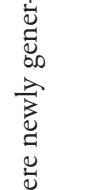 & 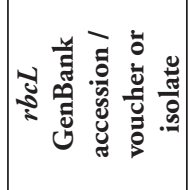 & 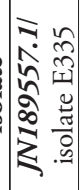 & 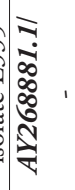 & 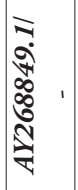 & 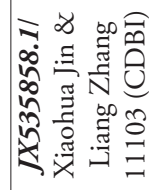 & 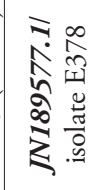 & 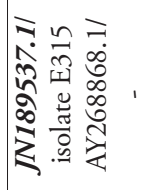 & 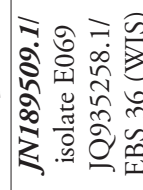 & 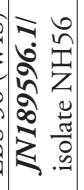 & 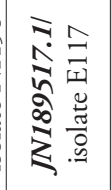 \\
\hline 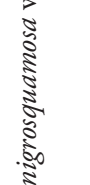 & 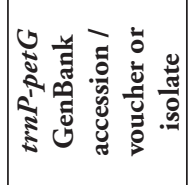 & 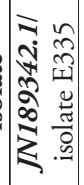 & 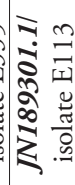 & 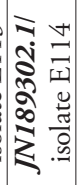 & 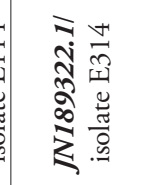 & 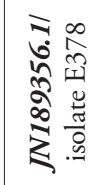 & 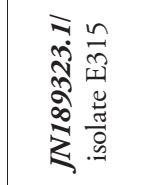 & 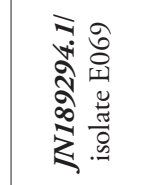 & 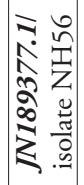 & 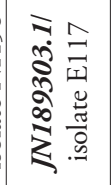 \\
\hline $\begin{array}{l}0 \\
0 \\
0 \\
\vdots \\
0 \\
0 \\
5 \\
5 \\
0 \\
0 \\
0 \\
0 \\
0 \\
0 \\
0\end{array}$ & 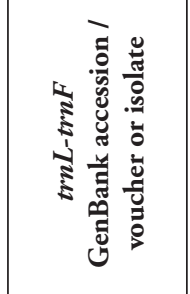 & 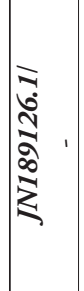 & 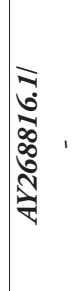 & 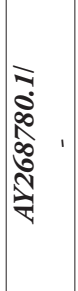 & 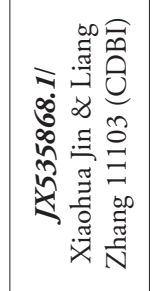 & 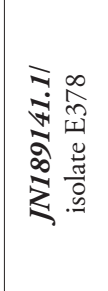 & 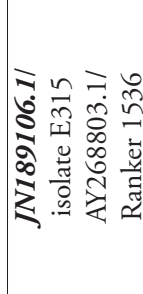 & 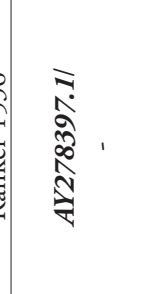 & 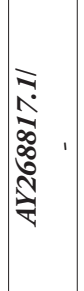 & 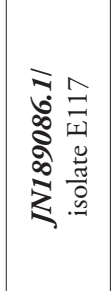 \\
\hline 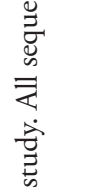 & 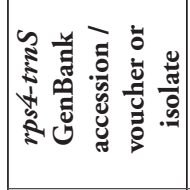 & 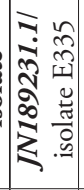 & 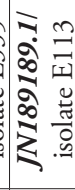 & 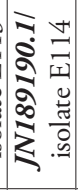 & 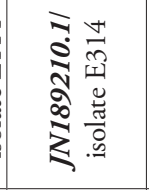 & 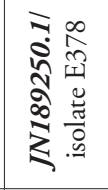 & 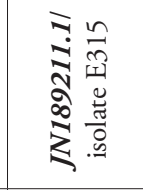 & 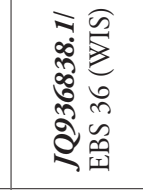 & 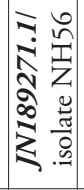 & 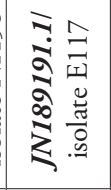 \\
\hline 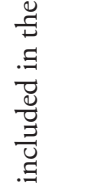 & 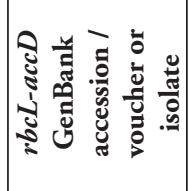 & 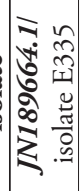 & 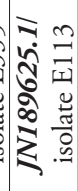 & 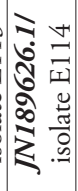 & 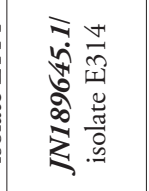 & 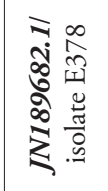 & 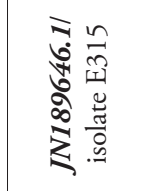 & 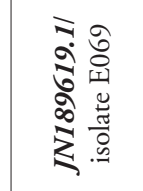 & 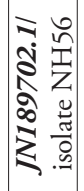 & 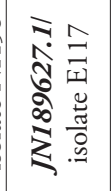 \\
\hline 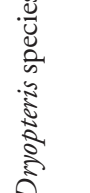 & 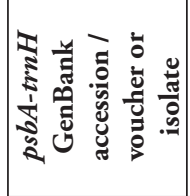 & 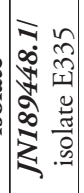 & 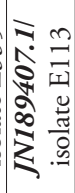 & 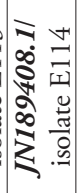 & 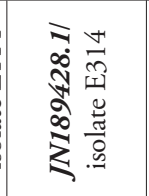 & 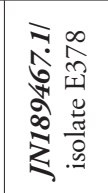 & 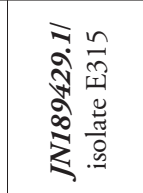 & 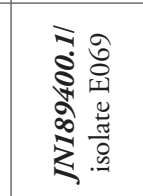 & 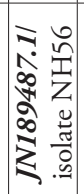 & 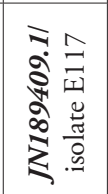 \\
\hline 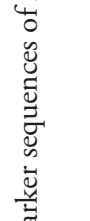 & 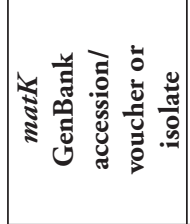 & & & & 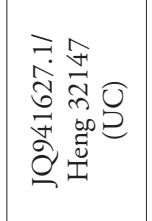 & 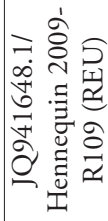 & 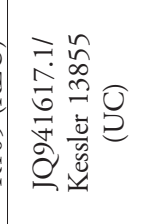 & 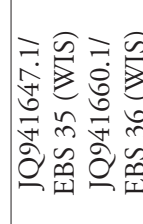 & & 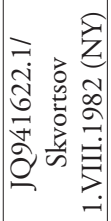 \\
\hline 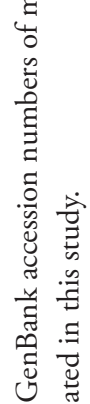 & 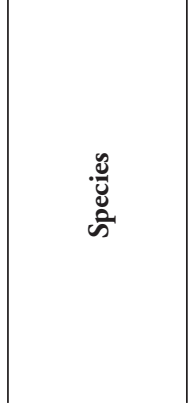 & 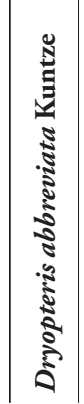 & 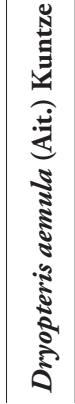 & 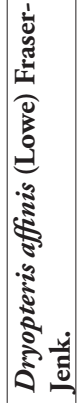 & 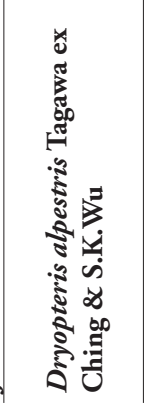 & 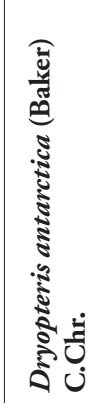 & 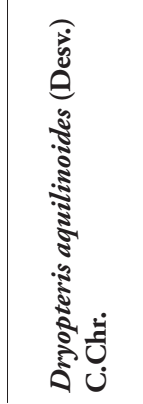 & 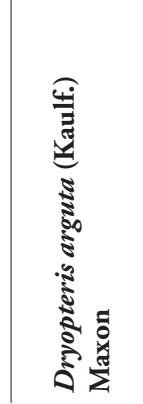 & 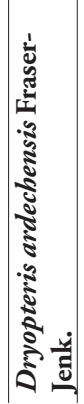 & 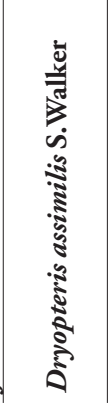 \\
\hline
\end{tabular}




\begin{tabular}{|c|c|c|c|c|c|c|c|}
\hline 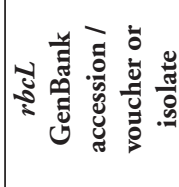 & 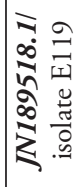 & 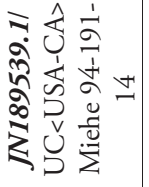 & 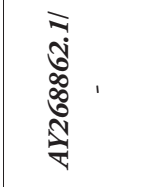 & 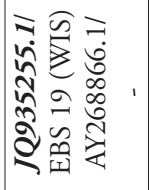 & 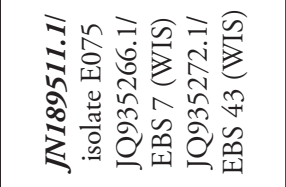 & 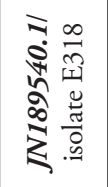 & 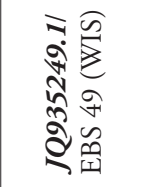 \\
\hline 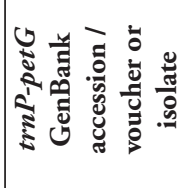 & 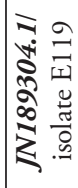 & 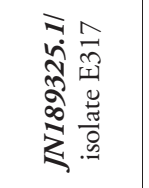 & 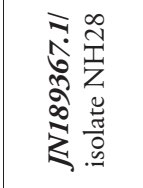 & 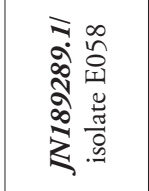 & 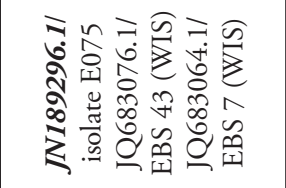 & 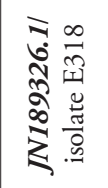 & 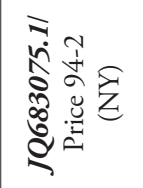 \\
\hline 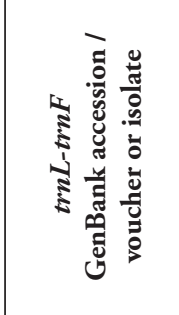 & 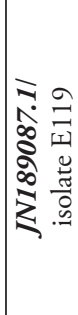 & 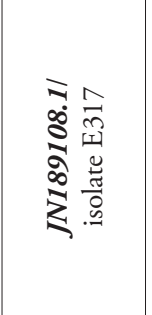 & 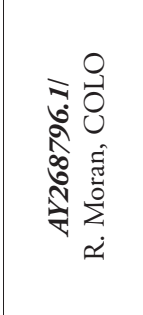 & 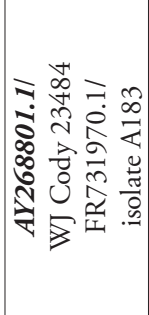 & 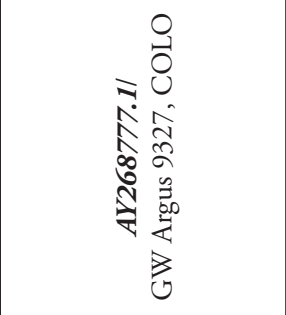 & 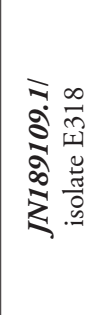 & 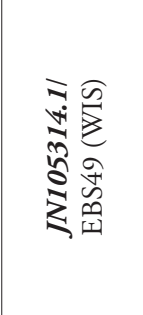 \\
\hline 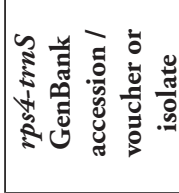 & 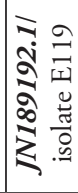 & 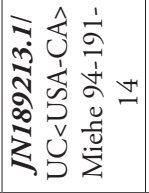 & 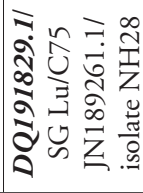 & 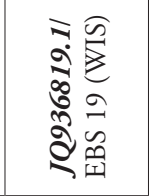 & 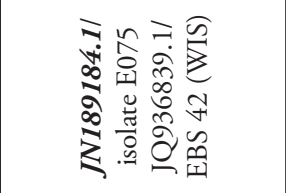 & 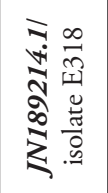 & 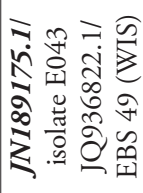 \\
\hline 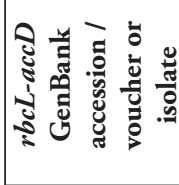 & 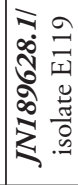 & 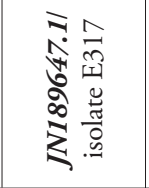 & 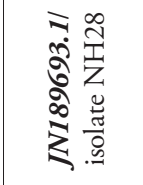 & 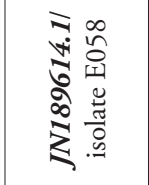 & 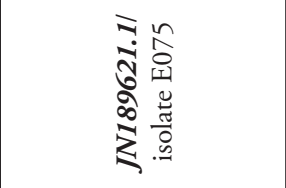 & 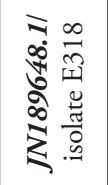 & 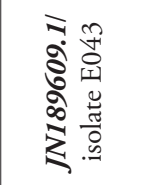 \\
\hline 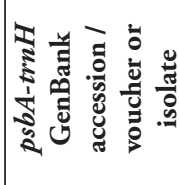 & $\begin{array}{ll}\nwarrow & 9 \\
0 & = \\
\pi & =1 \\
0 & 0 \\
0 & 0 \\
\vdots & 0\end{array}$ & 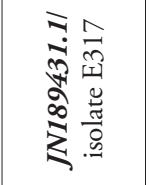 & 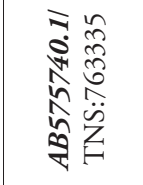 & 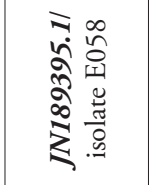 & 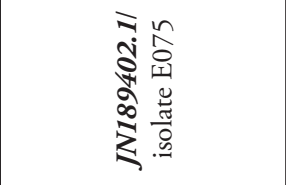 & 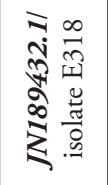 & 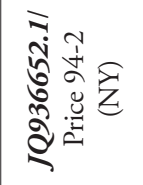 \\
\hline 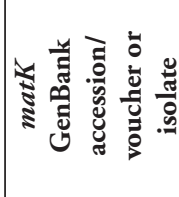 & 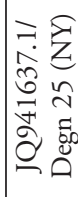 & & & 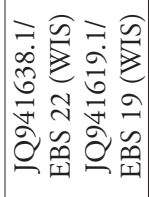 & 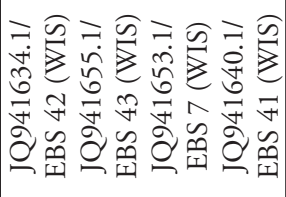 & 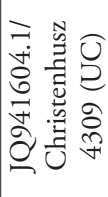 & 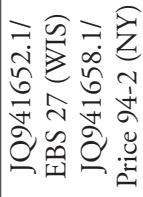 \\
\hline $\begin{array}{l}\text {. } \\
\text { कूँ } \\
\text { के }\end{array}$ & 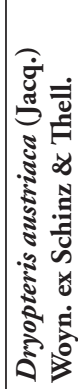 & 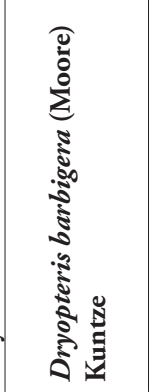 & 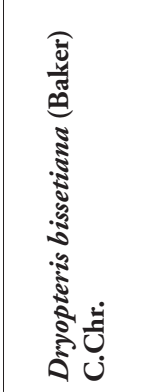 & 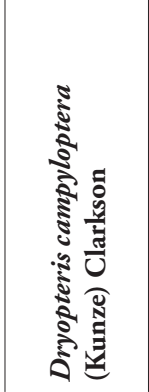 & 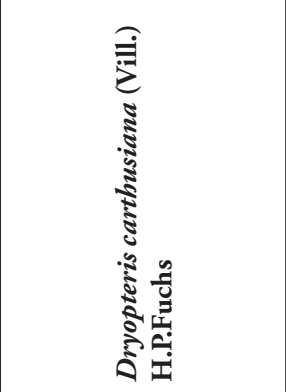 & 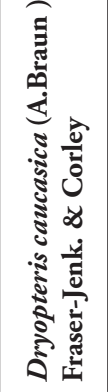 & 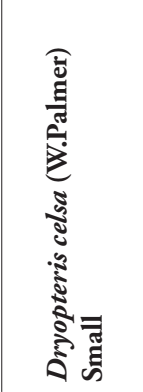 \\
\hline
\end{tabular}




\begin{tabular}{|c|c|c|c|c|c|c|c|}
\hline 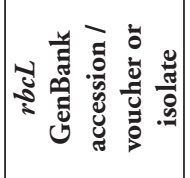 & 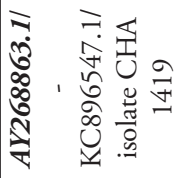 & 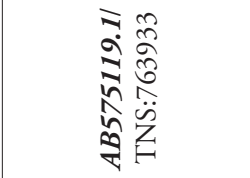 & 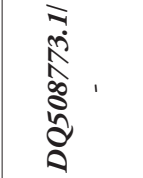 & 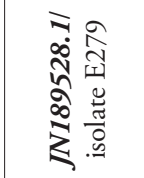 & 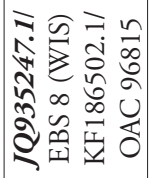 & 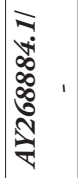 & 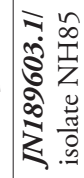 \\
\hline 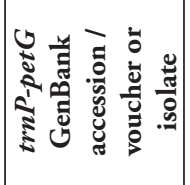 & 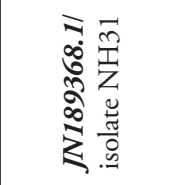 & 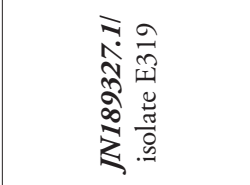 & 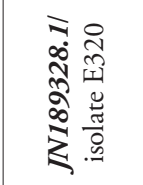 & 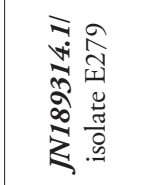 & 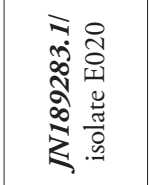 & 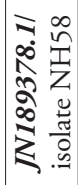 & 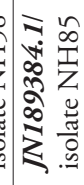 \\
\hline 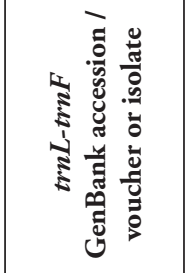 & 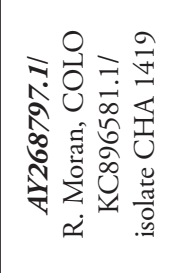 & 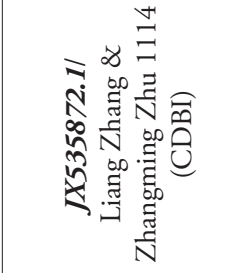 & 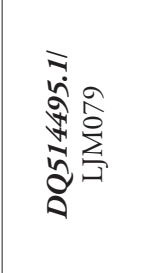 & 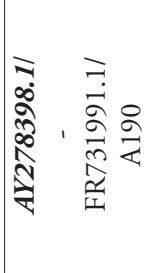 & 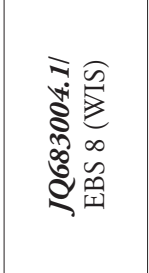 & 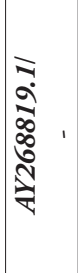 & 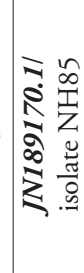 \\
\hline 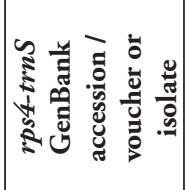 & 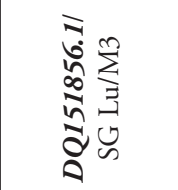 & 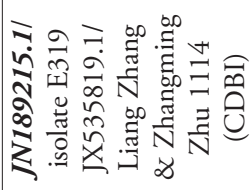 & 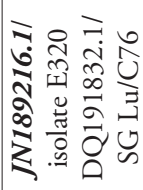 & 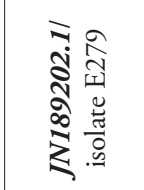 & 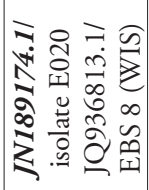 & 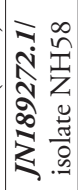 & 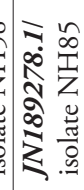 \\
\hline 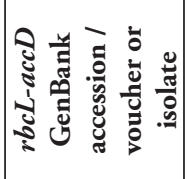 & 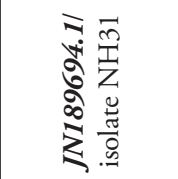 & 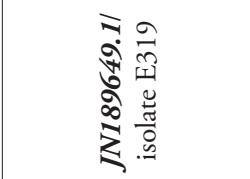 & 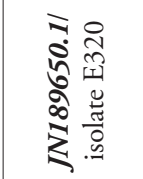 & 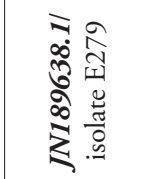 & 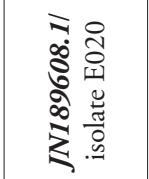 & 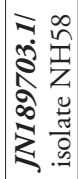 & 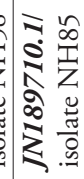 \\
\hline 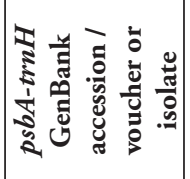 & 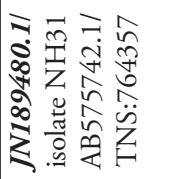 & 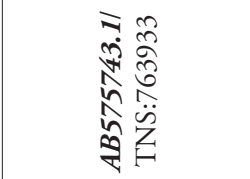 & 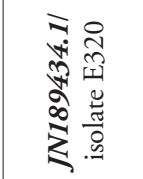 & 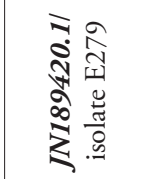 & 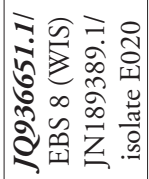 & 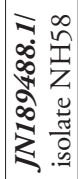 & 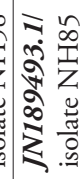 \\
\hline 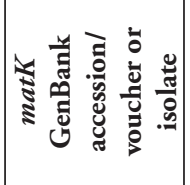 & & & & & 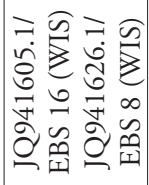 & 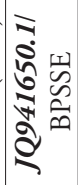 & \\
\hline 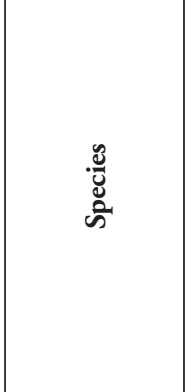 & 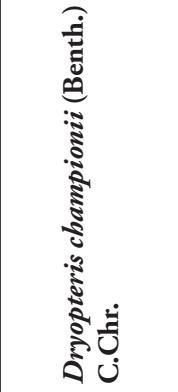 & 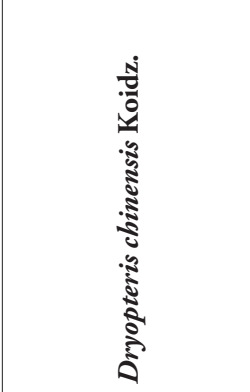 & 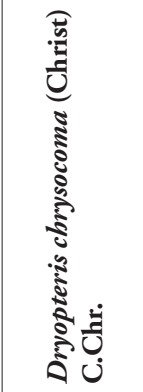 & 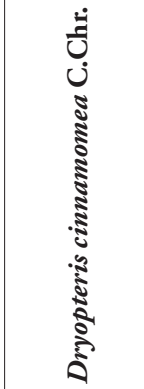 & 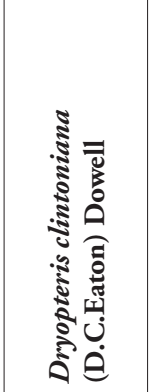 & 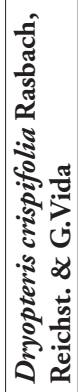 & 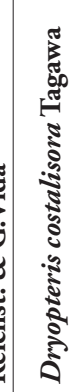 \\
\hline
\end{tabular}




\begin{tabular}{|c|c|c|}
\hline 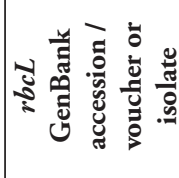 & 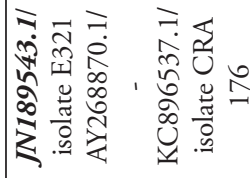 & 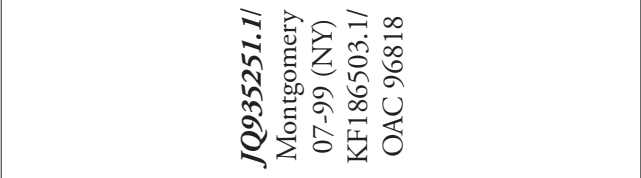 \\
\hline 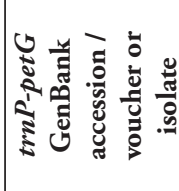 & 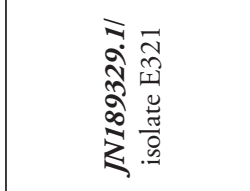 & 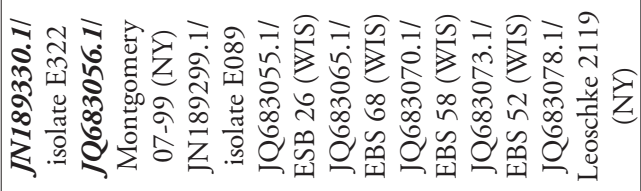 \\
\hline 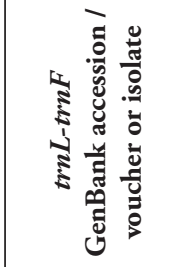 & 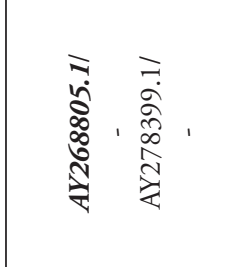 & 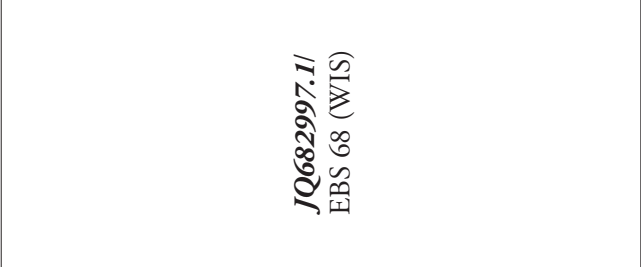 \\
\hline 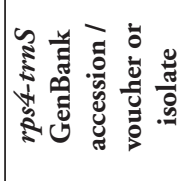 & 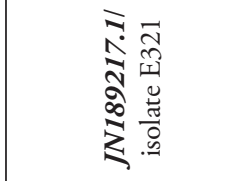 & 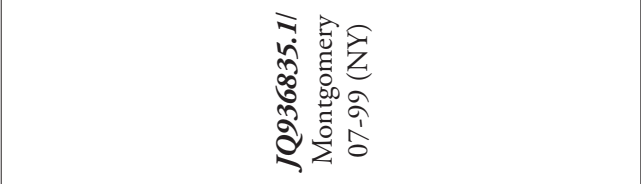 \\
\hline 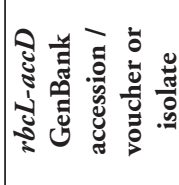 & 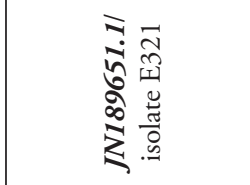 & 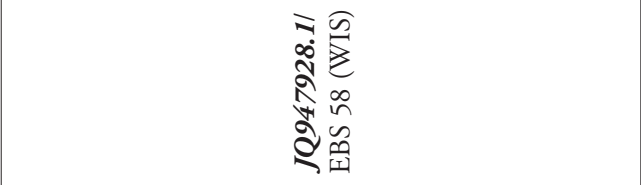 \\
\hline 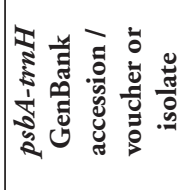 & 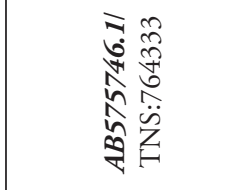 & 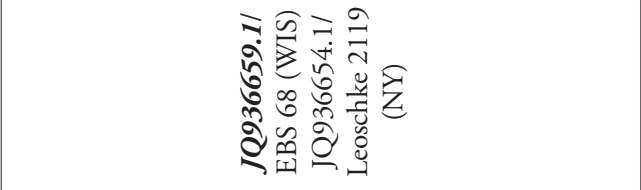 \\
\hline 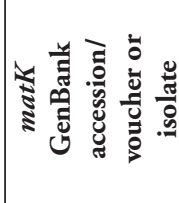 & & 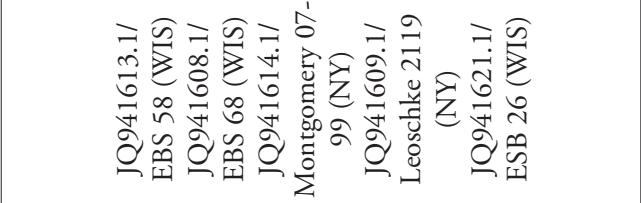 \\
\hline 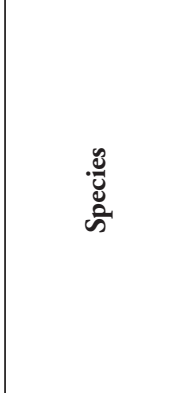 & 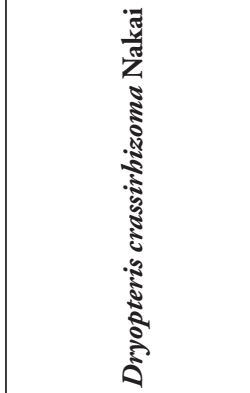 & 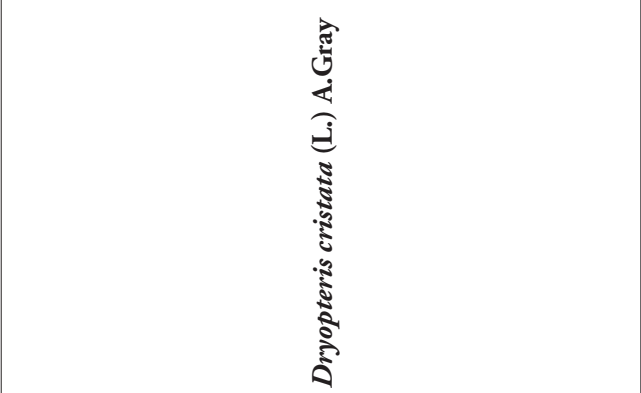 \\
\hline
\end{tabular}




\begin{tabular}{|c|c|c|c|c|c|}
\hline 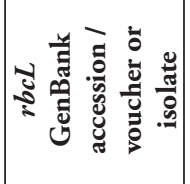 & 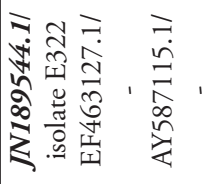 & 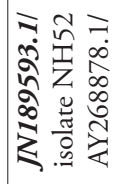 & 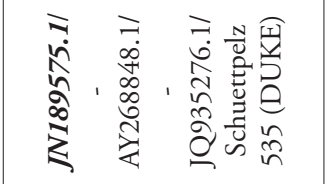 & 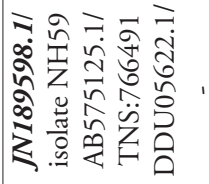 & \\
\hline 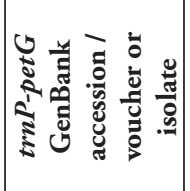 & 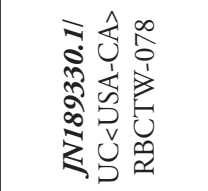 & 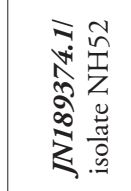 & 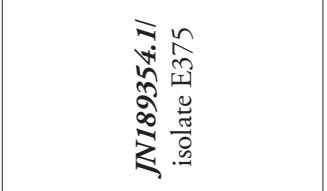 & 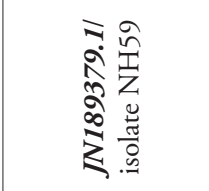 & \\
\hline 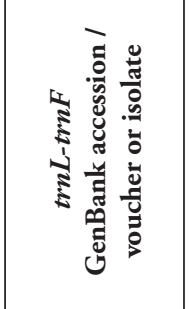 & 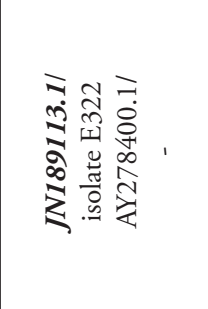 & 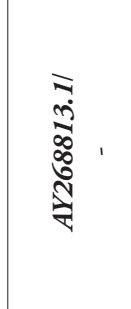 & 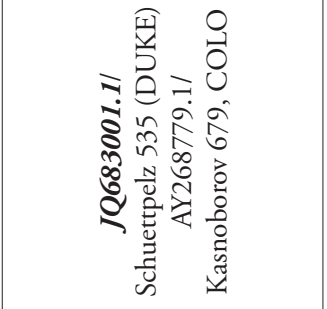 & 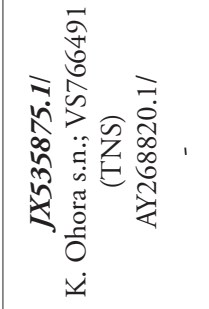 & \\
\hline 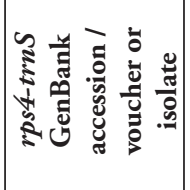 & 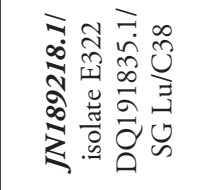 & 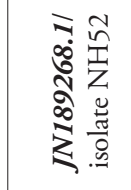 & 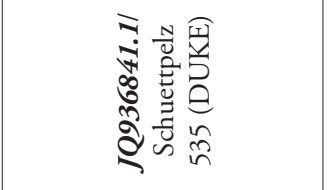 & 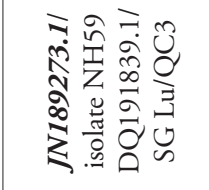 & 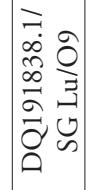 \\
\hline 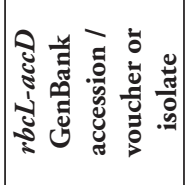 & 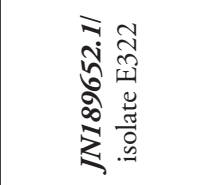 & 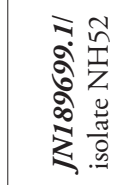 & 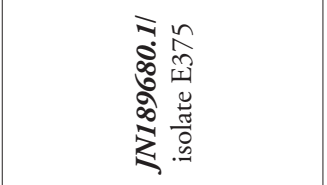 & 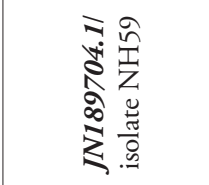 & \\
\hline 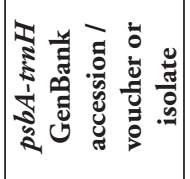 & 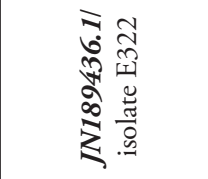 & 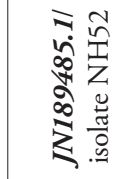 & 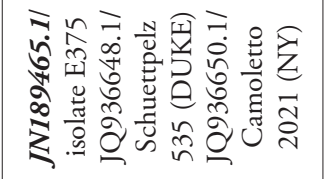 & 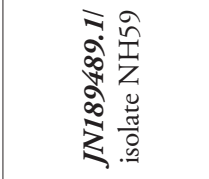 & \\
\hline 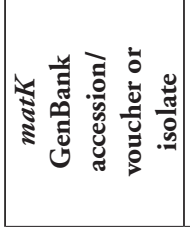 & & & 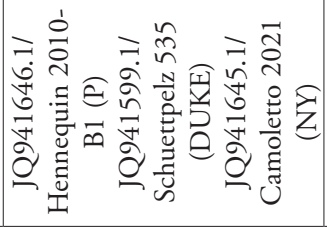 & & \\
\hline 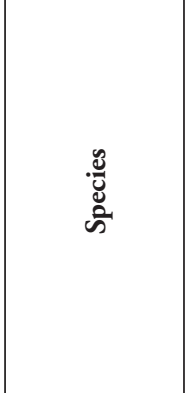 & 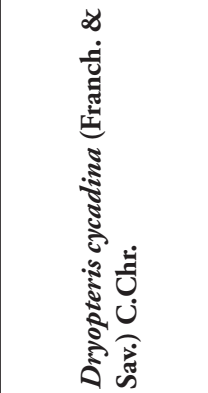 & 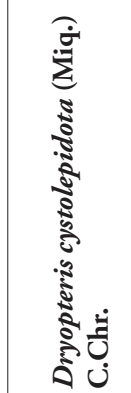 & 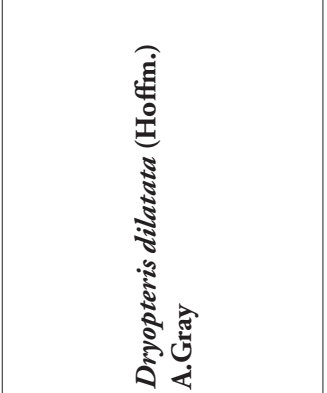 & 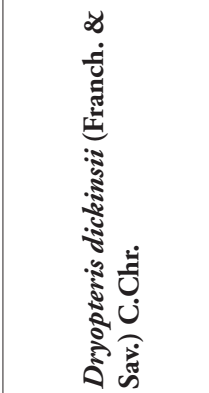 & 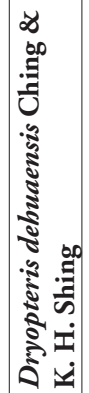 \\
\hline
\end{tabular}




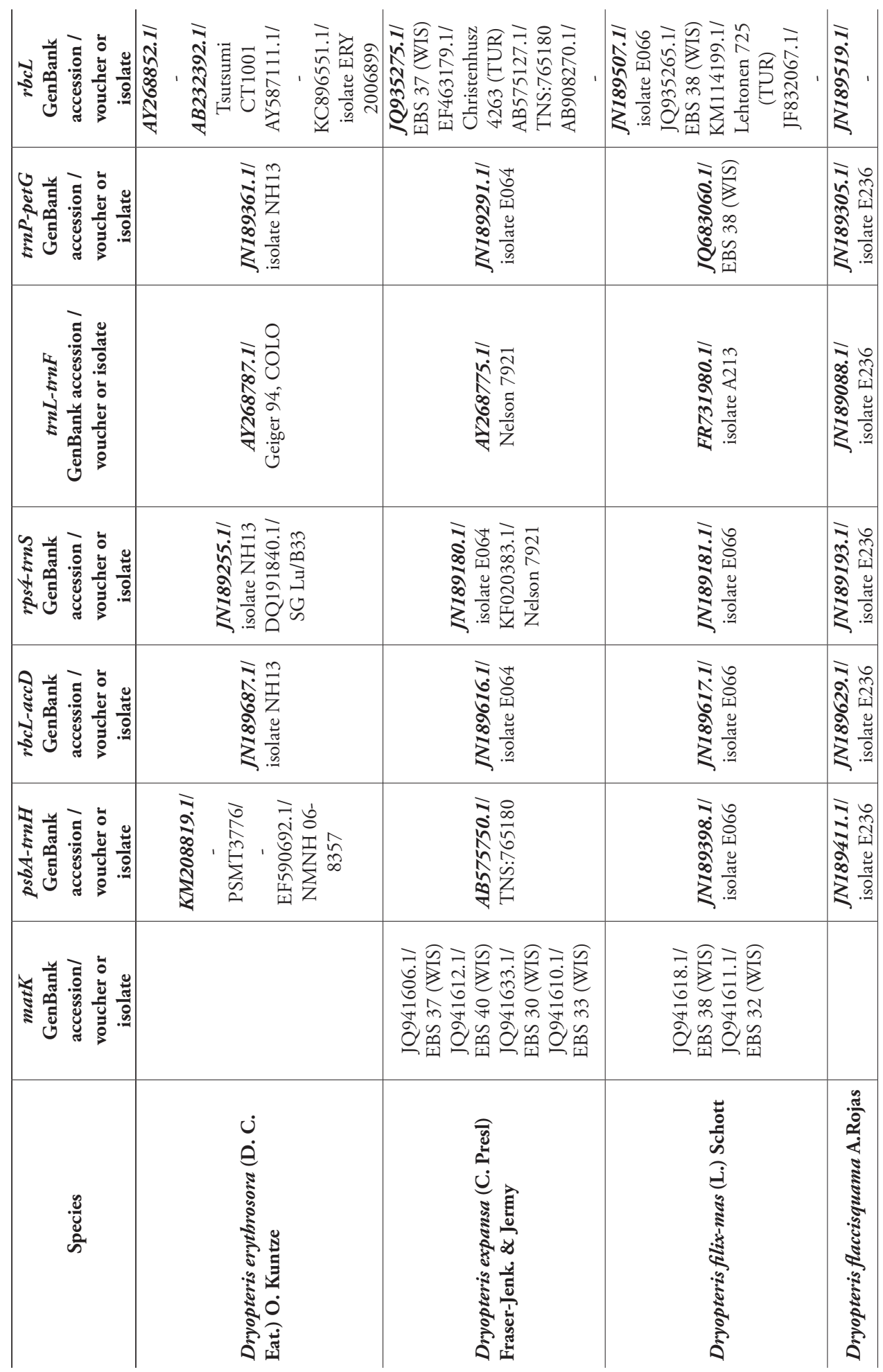




\begin{tabular}{|c|c|c|c|c|c|}
\hline 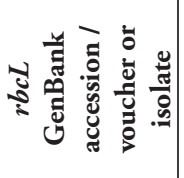 & 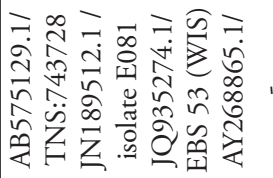 & 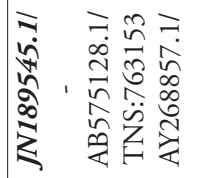 & 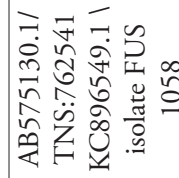 & 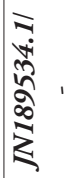 & 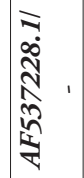 \\
\hline 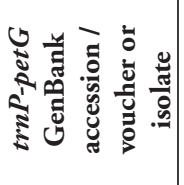 & 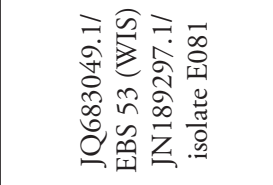 & 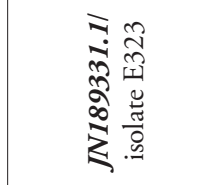 & & 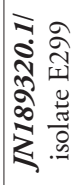 & 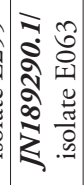 \\
\hline 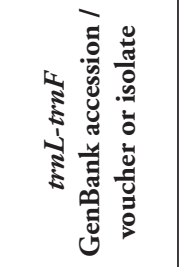 & 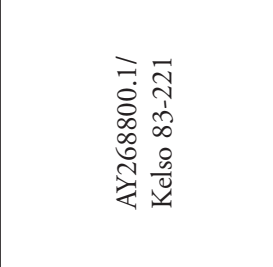 & 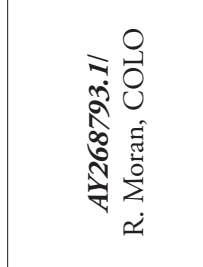 & $\begin{array}{l}7 \\
\infty \\
\infty \\
0 \\
0 \\
0 \\
\infty \\
0 \\
0 \\
0 \\
0\end{array}$ & 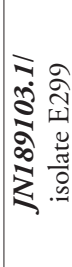 & 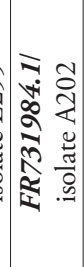 \\
\hline 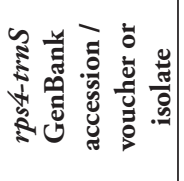 & 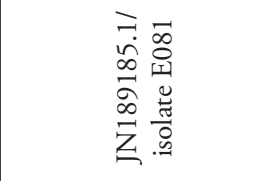 & 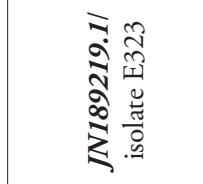 & 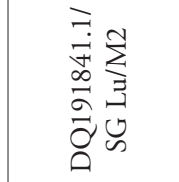 & 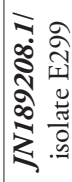 & को \\
\hline 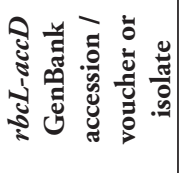 & & 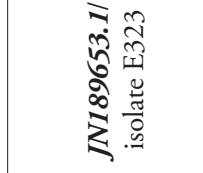 & & 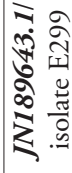 & 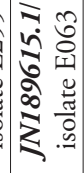 \\
\hline 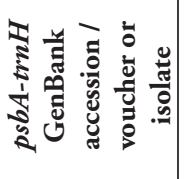 & 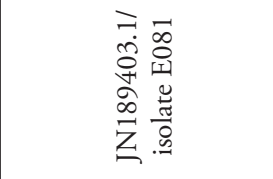 & 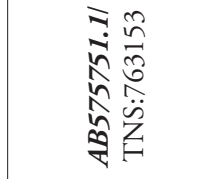 & 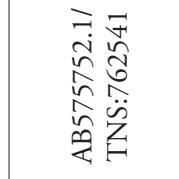 & 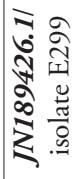 & 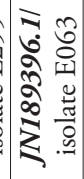 \\
\hline 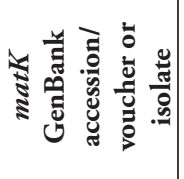 & 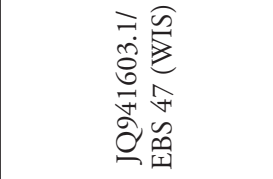 & & & & \\
\hline $\begin{array}{l}\frac{8}{\tilde{J}} \\
\text { के }\end{array}$ & 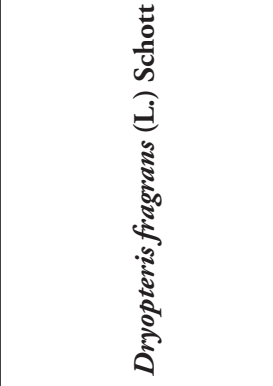 & 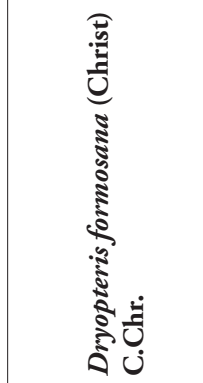 & 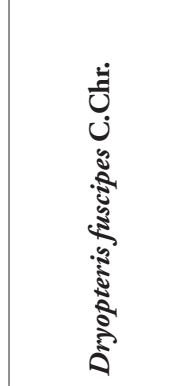 & 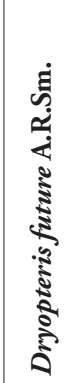 & 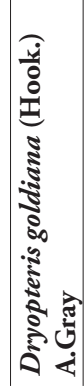 \\
\hline
\end{tabular}




\begin{tabular}{|c|c|c|c|c|c|c|}
\hline 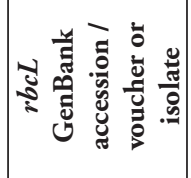 & 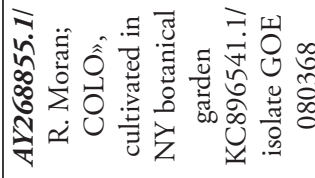 & 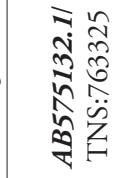 & 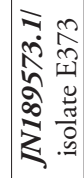 & 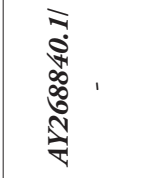 & 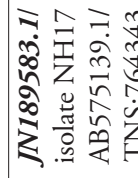 & 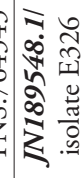 \\
\hline 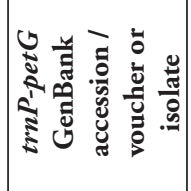 & 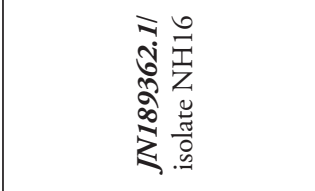 & 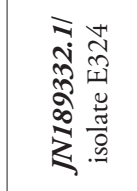 & 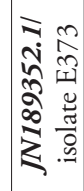 & 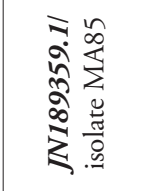 & 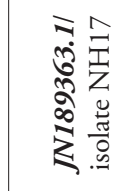 & 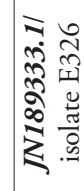 \\
\hline 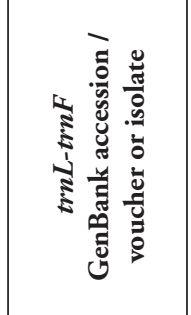 & 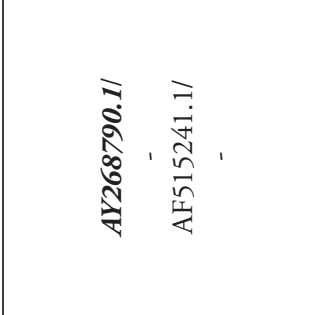 & 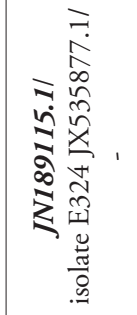 & 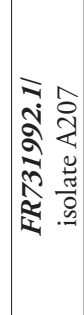 & 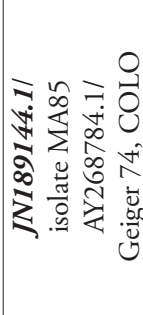 & 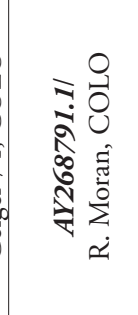 & 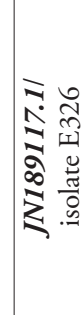 \\
\hline 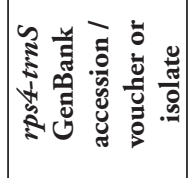 & 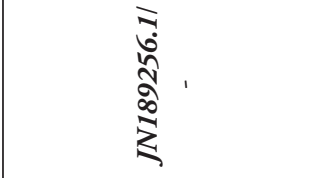 & 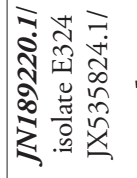 & 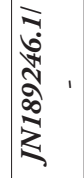 & 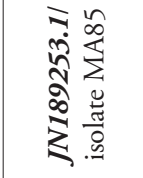 & 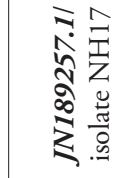 & 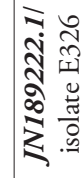 \\
\hline 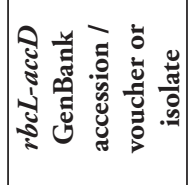 & 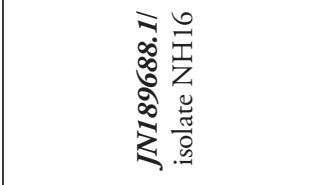 & 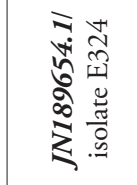 & $\begin{array}{ll}\overline{1} & 0 \\
0 & 0 \\
0 & 0 \\
0 & =1 \\
0 & 0 \\
0 & 0 \\
\vdots & 0\end{array}$ & $\begin{array}{l}\overline{1} \\
1 \\
0 \\
0 \\
0 \\
\infty \\
\infty \\
\vdots \\
\vdots \\
\vdots \\
\vdots\end{array}$ & 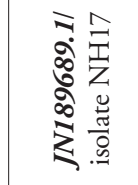 & 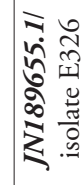 \\
\hline 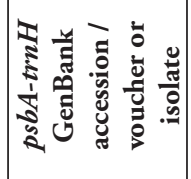 & 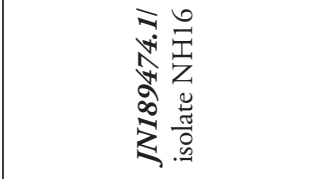 & 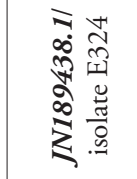 & 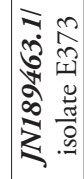 & 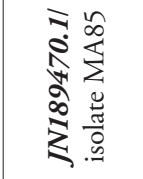 & 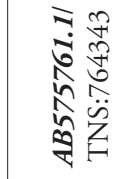 & 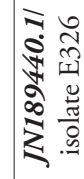 \\
\hline 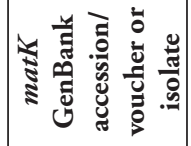 & & & & & & \\
\hline 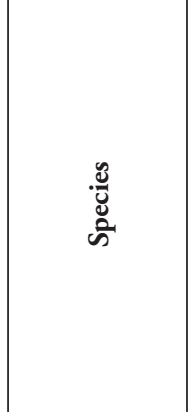 & 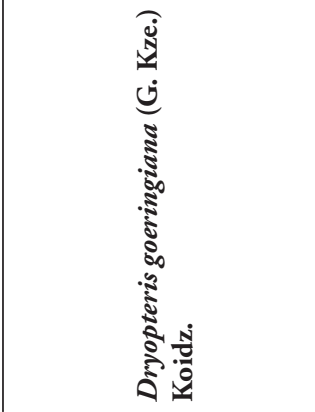 & 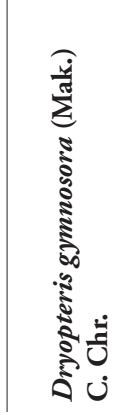 & 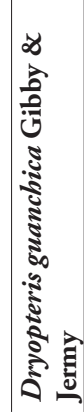 & 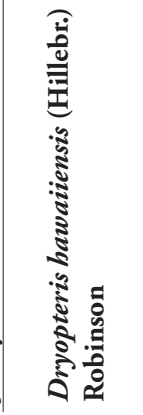 & 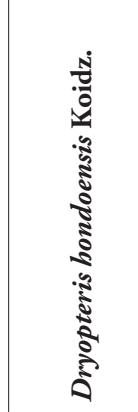 & 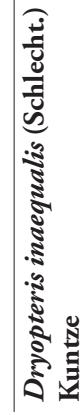 \\
\hline
\end{tabular}




\begin{tabular}{|c|c|c|c|c|c|c|c|}
\hline 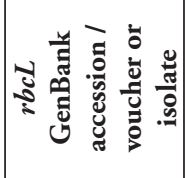 & 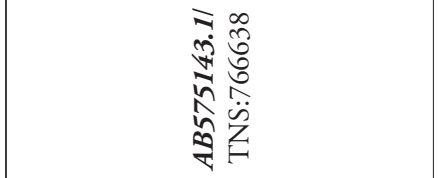 & 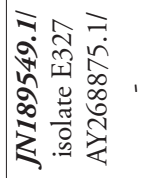 & 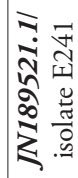 & 命 & 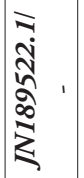 & 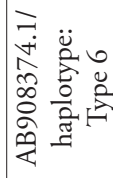 & 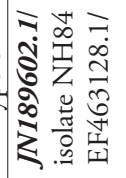 \\
\hline 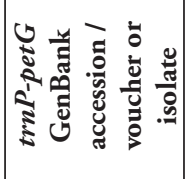 & 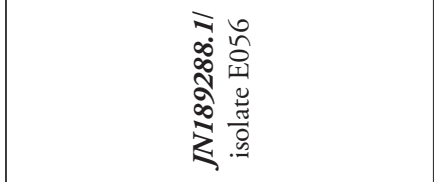 & 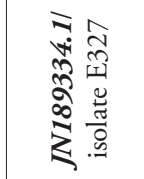 & 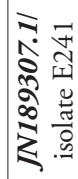 & 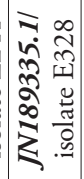 & 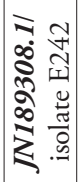 & & 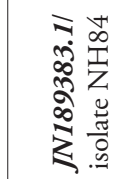 \\
\hline 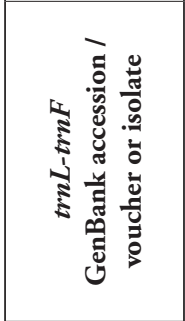 & 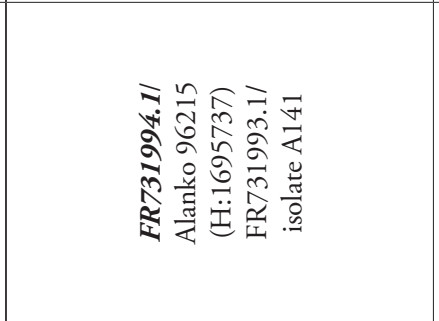 & 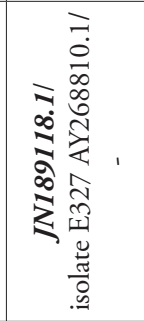 & 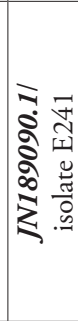 & 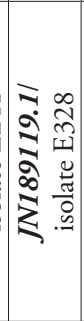 & 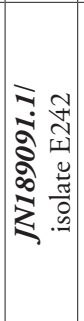 & & 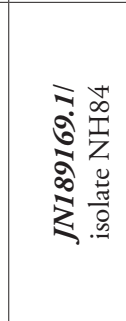 \\
\hline 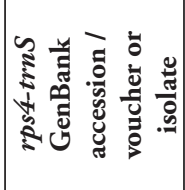 & 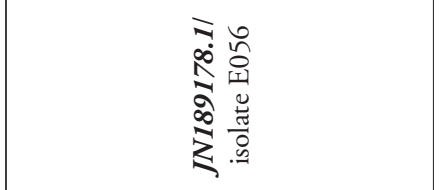 & 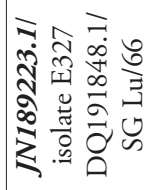 & 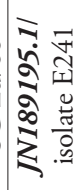 & 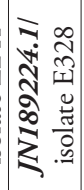 & 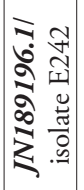 & & 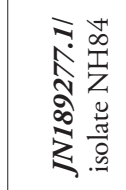 \\
\hline 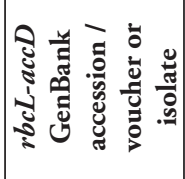 & 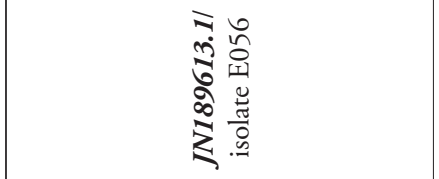 & 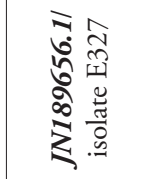 & 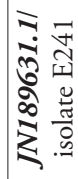 & 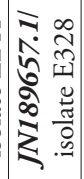 & 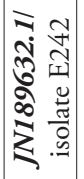 & & 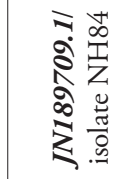 \\
\hline 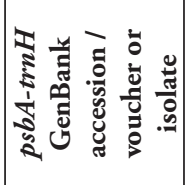 & 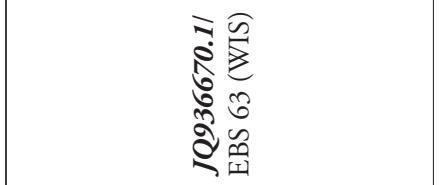 & 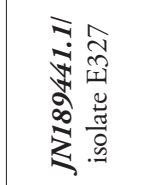 & 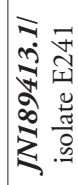 & 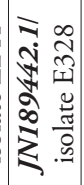 & 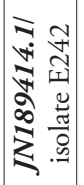 & & 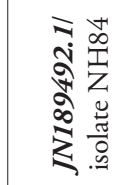 \\
\hline 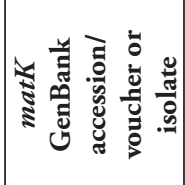 & 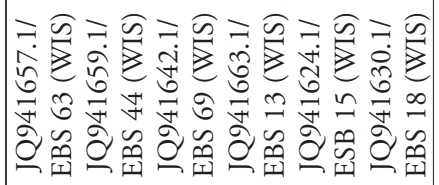 & & & & & & \\
\hline $\begin{array}{l}\stackrel{\mathscr{U}}{u} \\
\text { के }\end{array}$ & 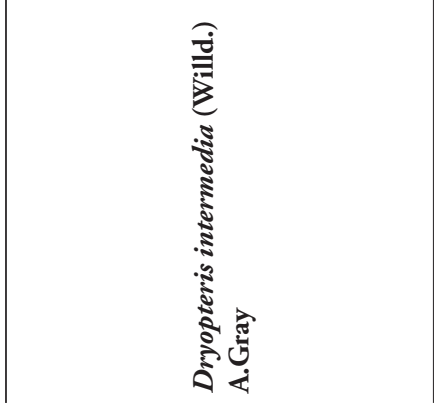 & 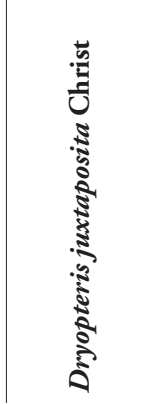 & 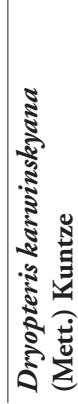 & 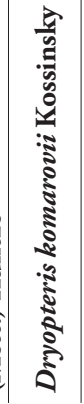 & 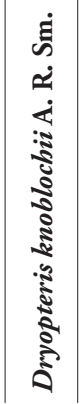 & 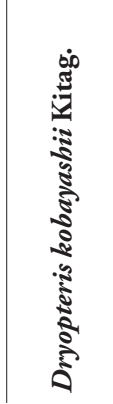 & 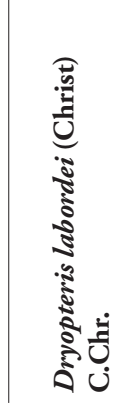 \\
\hline
\end{tabular}




\begin{tabular}{|c|c|c|c|c|c|c|c|}
\hline 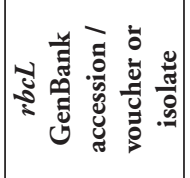 & 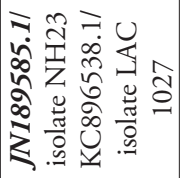 & 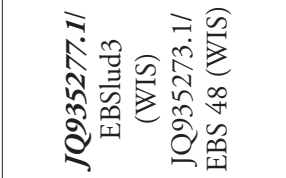 & 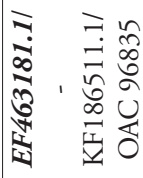 & 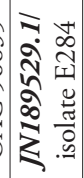 & 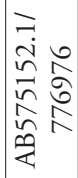 & 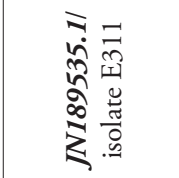 & 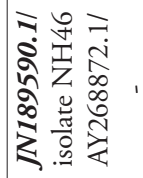 \\
\hline 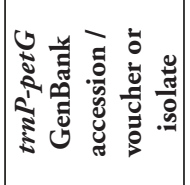 & 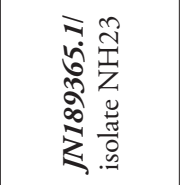 & 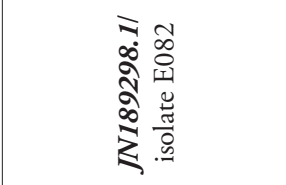 & 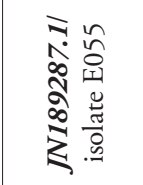 & 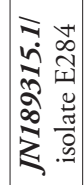 & & 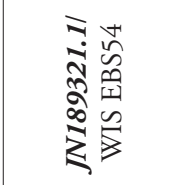 & 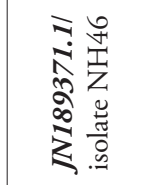 \\
\hline 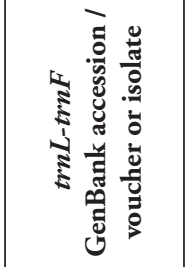 & 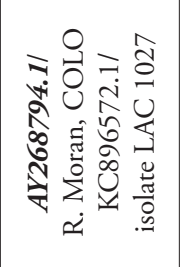 & 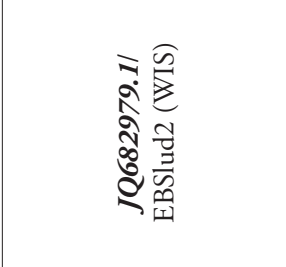 & 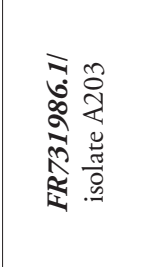 & 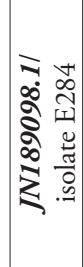 & & 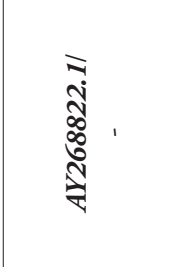 & 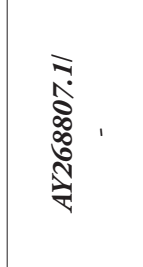 \\
\hline 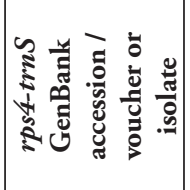 & 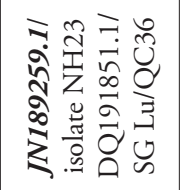 & 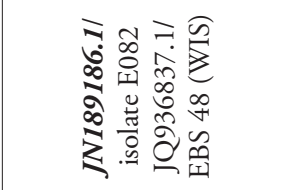 & 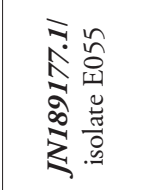 & 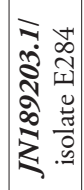 & & 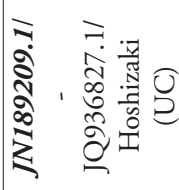 & 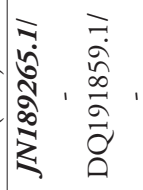 \\
\hline 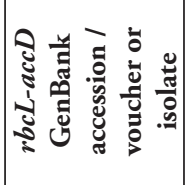 & 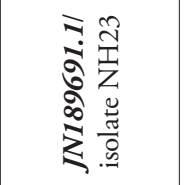 & 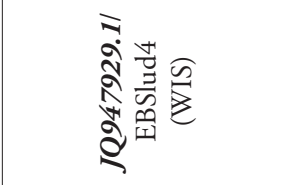 & 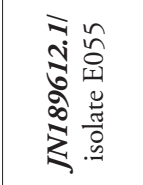 & 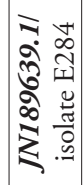 & & 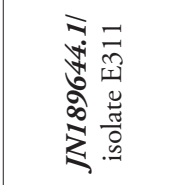 & 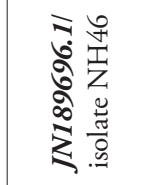 \\
\hline 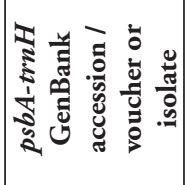 & 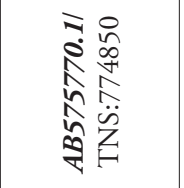 & 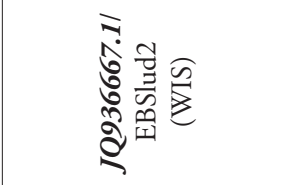 & 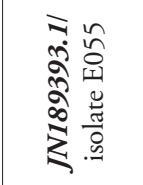 & 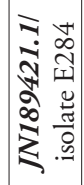 & 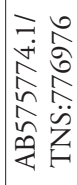 & 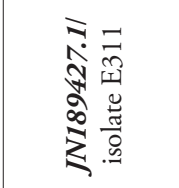 & 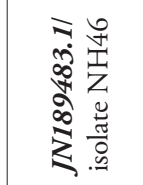 \\
\hline 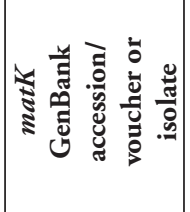 & & 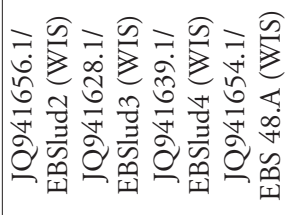 & 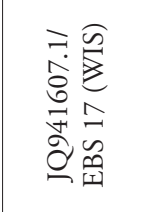 & & & 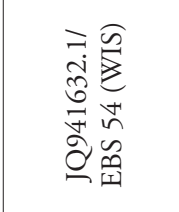 & \\
\hline 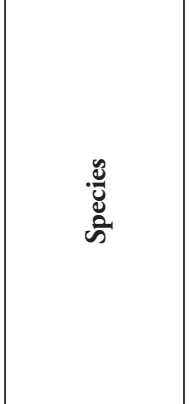 & 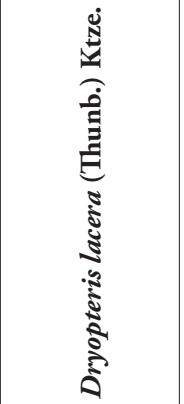 & 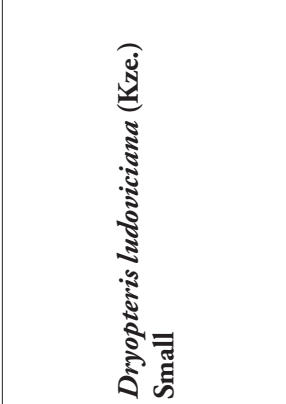 & 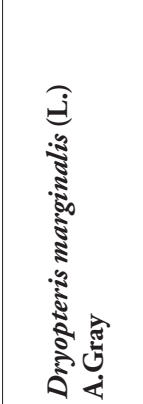 & 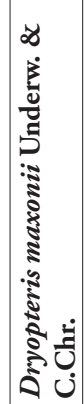 & 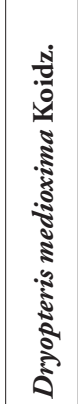 & 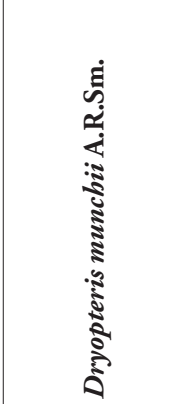 & 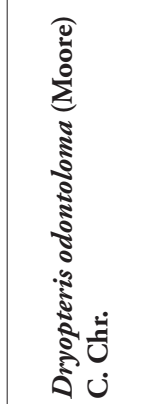 \\
\hline
\end{tabular}




\begin{tabular}{|c|c|c|c|c|c|c|c|c|c|}
\hline 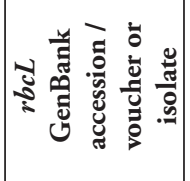 & 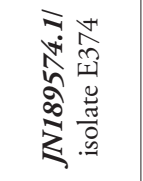 & 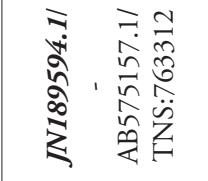 & 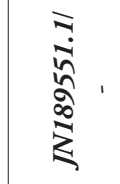 & 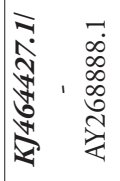 & 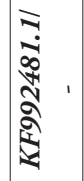 & 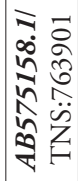 & 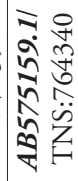 & 离 & 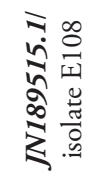 \\
\hline 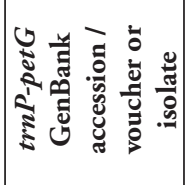 & 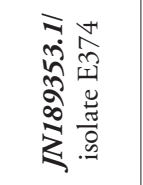 & 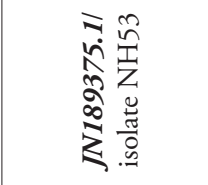 & 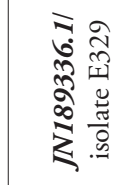 & 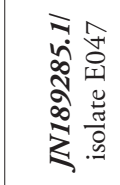 & 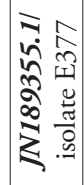 & 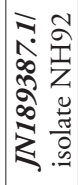 & 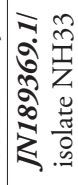 & 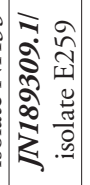 & 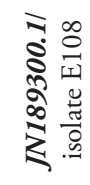 \\
\hline 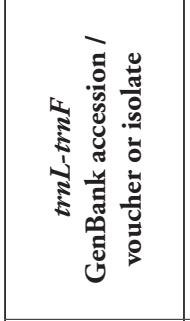 & 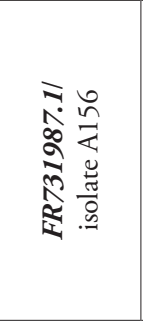 & 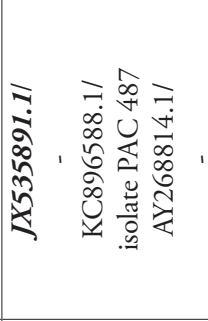 & 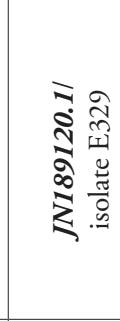 & 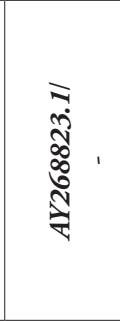 & 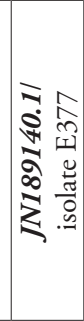 & 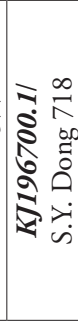 & 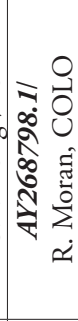 & $\frac{\sqrt{1}}{\sqrt{2}}$ & 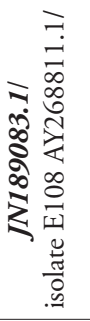 \\
\hline 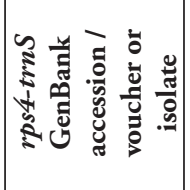 & 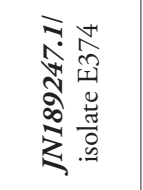 & 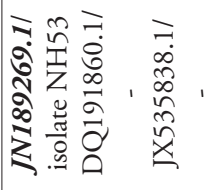 & 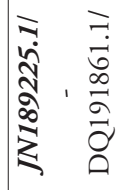 & 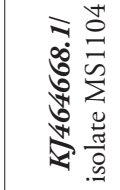 & 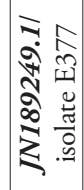 & 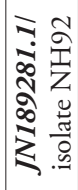 & 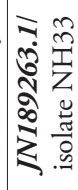 & 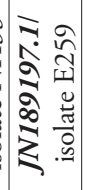 & 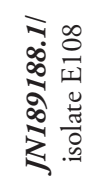 \\
\hline 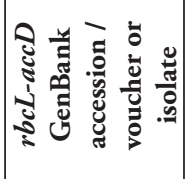 & 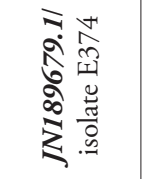 & 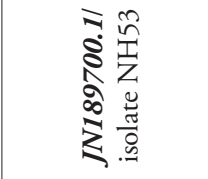 & 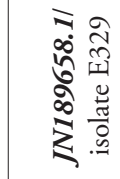 & 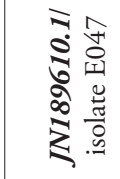 & 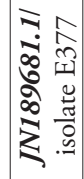 & 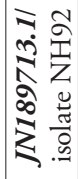 & 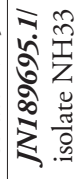 & 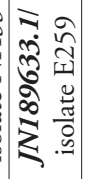 & 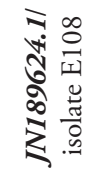 \\
\hline 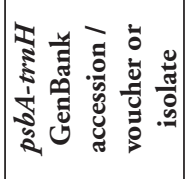 & 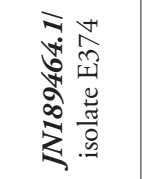 & 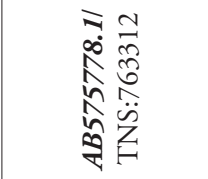 & 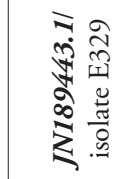 & 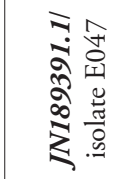 & 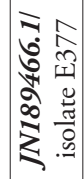 & 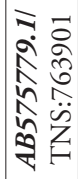 & 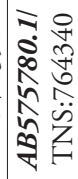 & 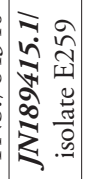 & 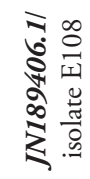 \\
\hline 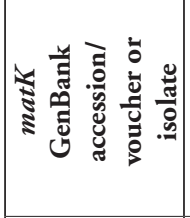 & 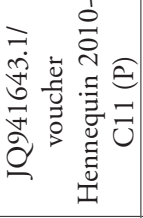 & & & & & 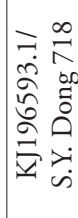 & & & \\
\hline $\begin{array}{l}\text {. } \\
\text { के } \\
\text { के }\end{array}$ & 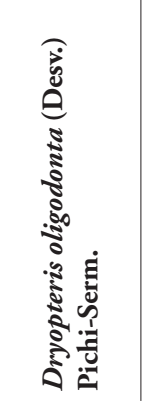 & 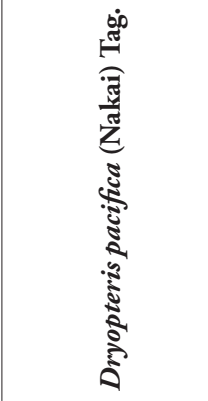 & 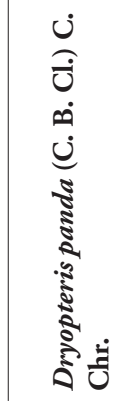 & 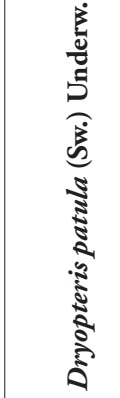 & 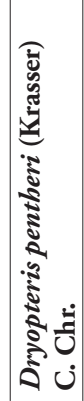 & 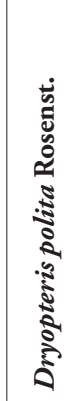 & 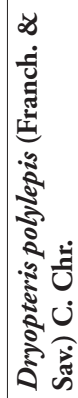 & 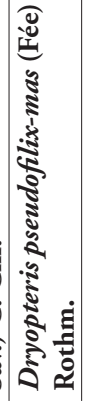 & 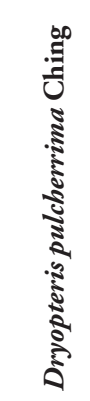 \\
\hline
\end{tabular}




\begin{tabular}{|c|c|c|c|c|c|c|c|c|}
\hline 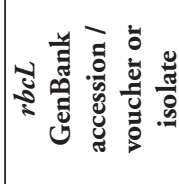 & 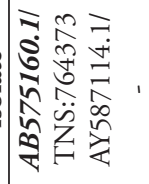 & 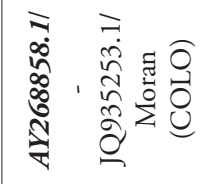 & 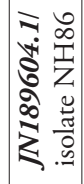 & ゙ָ & 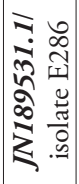 & 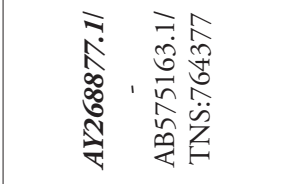 & 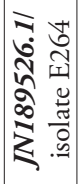 & 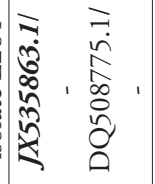 \\
\hline 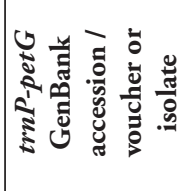 & 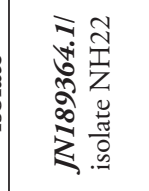 & 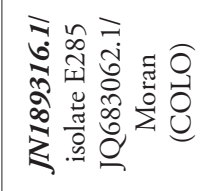 & 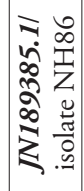 & 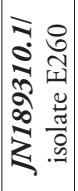 & 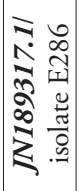 & 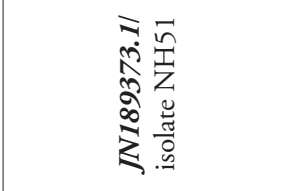 & 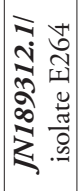 & 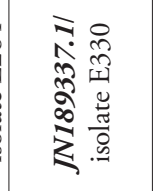 \\
\hline 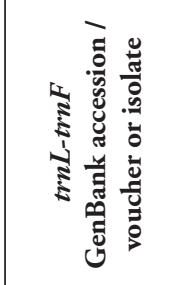 & 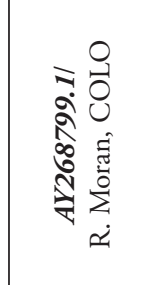 & 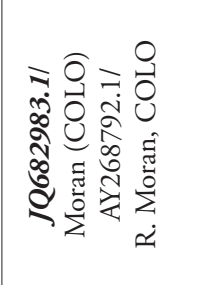 & 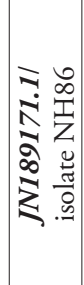 & 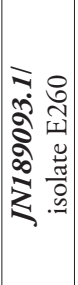 & 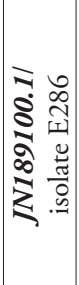 & 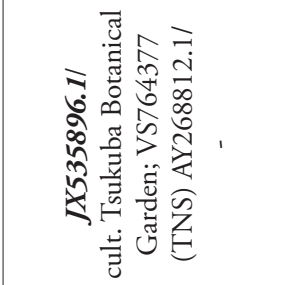 & 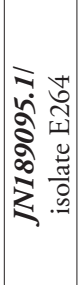 & 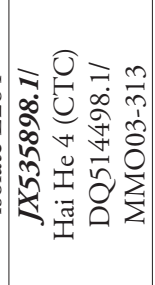 \\
\hline 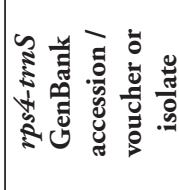 & 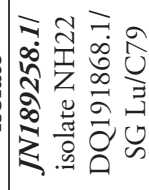 & 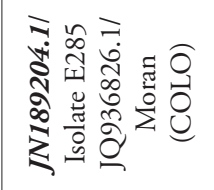 & 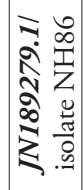 & 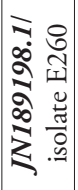 & 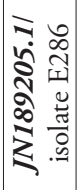 & 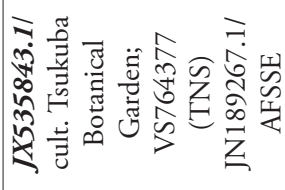 & 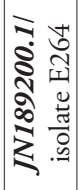 & 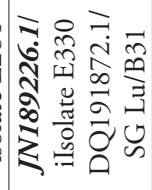 \\
\hline 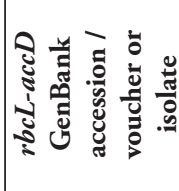 & 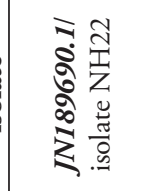 & 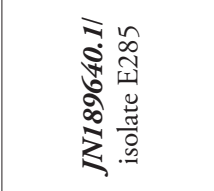 & 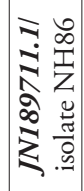 & 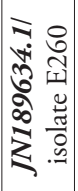 & 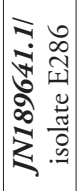 & 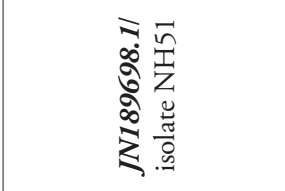 & 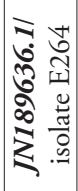 & 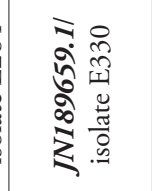 \\
\hline 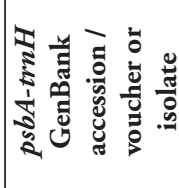 & 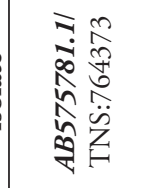 & 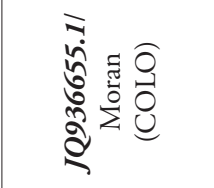 & 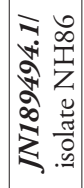 & 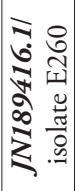 & 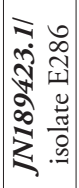 & 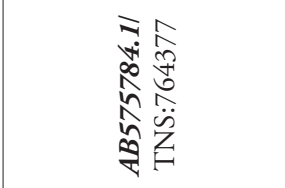 & 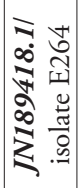 & 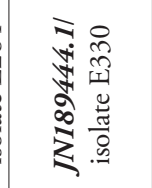 \\
\hline 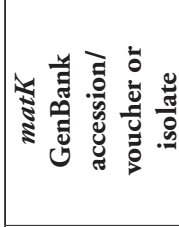 & & 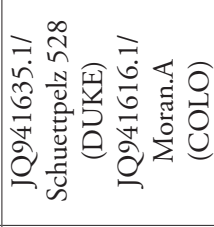 & & & & & & 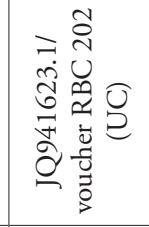 \\
\hline 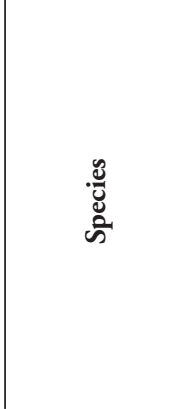 & 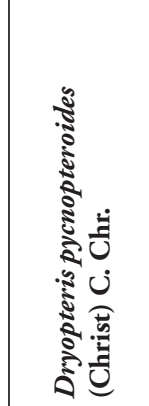 & 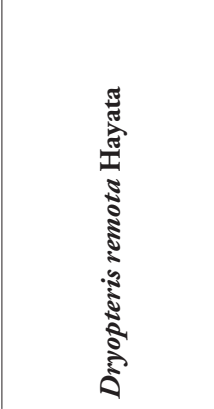 & 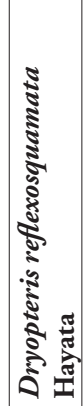 & 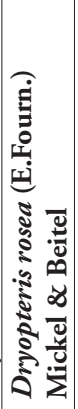 & 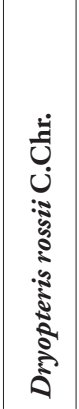 & 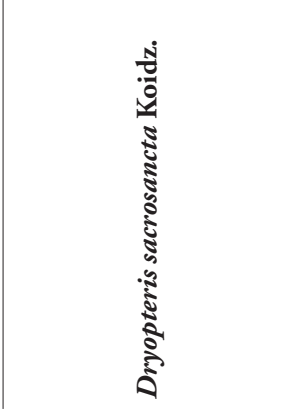 & 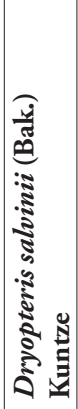 & 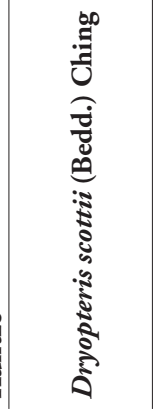 \\
\hline
\end{tabular}




\begin{tabular}{|c|c|c|c|c|c|c|c|c|}
\hline 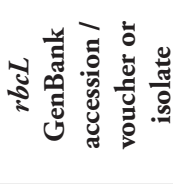 & 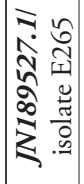 & 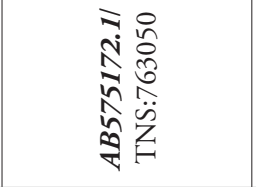 & 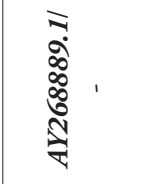 & 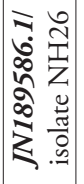 & 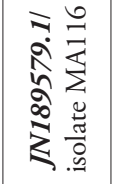 & 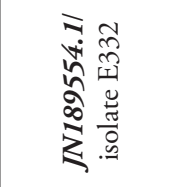 & 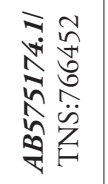 & 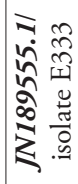 \\
\hline 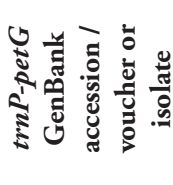 & 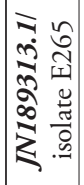 & 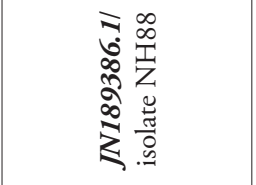 & 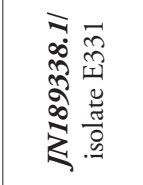 & 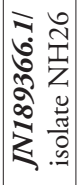 & 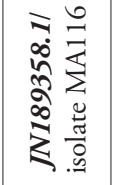 & 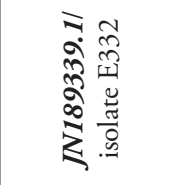 & 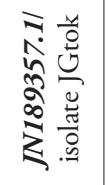 & 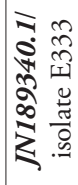 \\
\hline 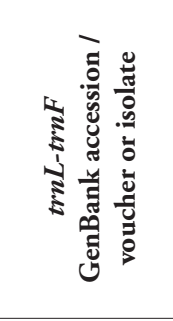 & 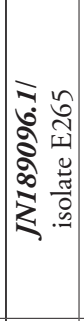 & 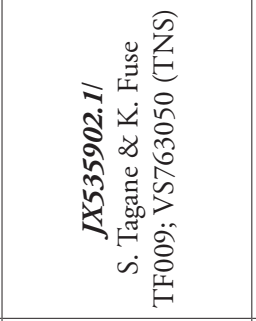 & 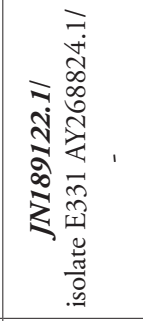 & 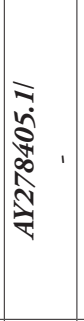 & 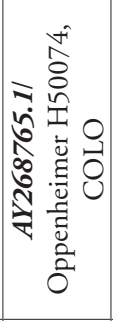 & 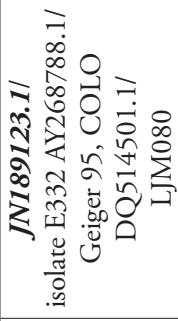 & 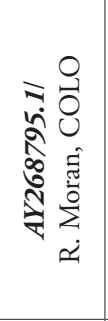 & 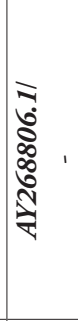 \\
\hline 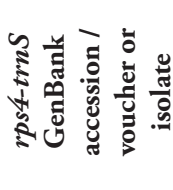 & 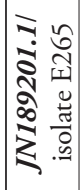 & 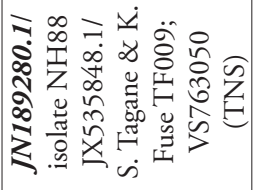 & 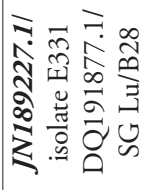 & 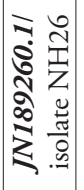 & 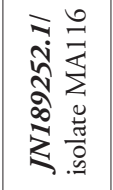 & 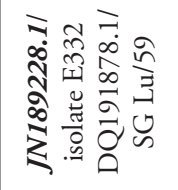 & 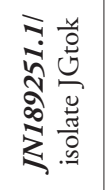 & 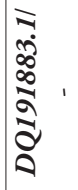 \\
\hline 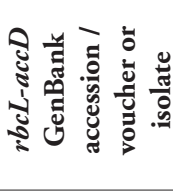 & 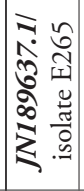 & 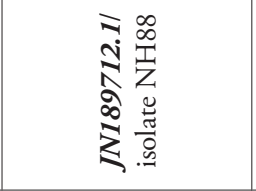 & 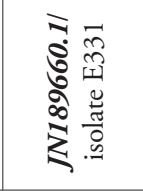 & 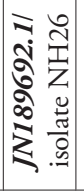 & 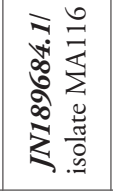 & 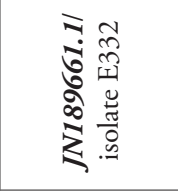 & 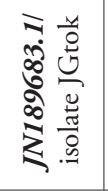 & 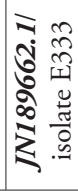 \\
\hline 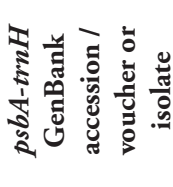 & 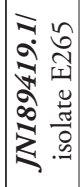 & 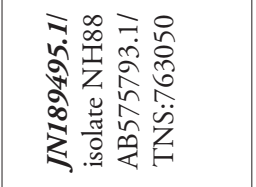 & 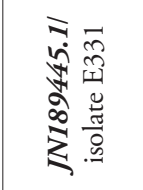 & 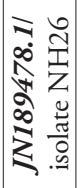 & 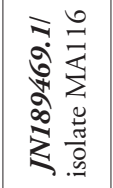 & 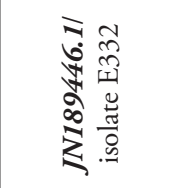 & 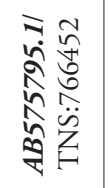 & 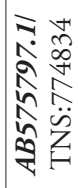 \\
\hline 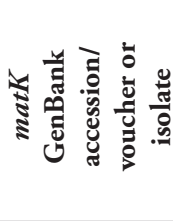 & & & & & & & 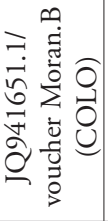 & \\
\hline $\begin{array}{l}\text { कू. } \\
\text { के } \\
\text { के }\end{array}$ & 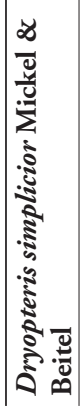 & 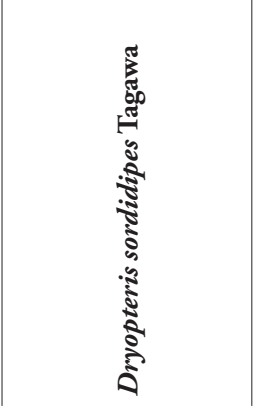 & 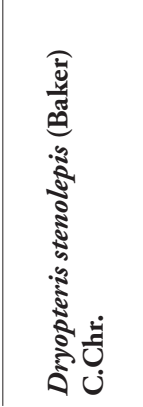 & 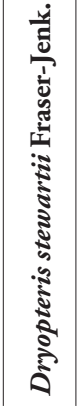 & 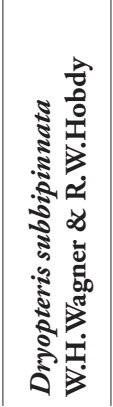 & 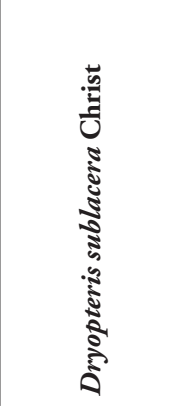 & 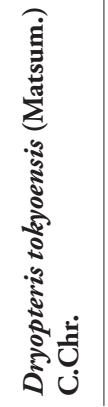 & 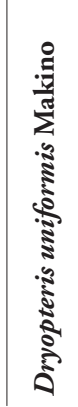 \\
\hline
\end{tabular}




\begin{tabular}{|c|c|c|c|c|c|}
\hline 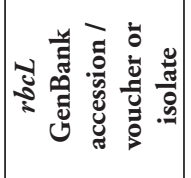 & 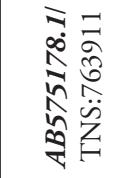 & 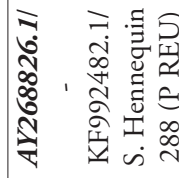 & 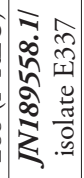 & 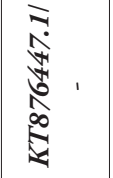 & 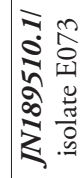 \\
\hline 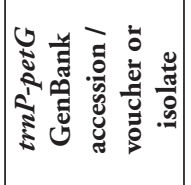 & 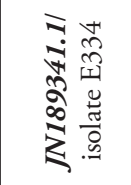 & 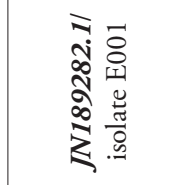 & 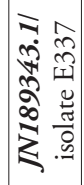 & 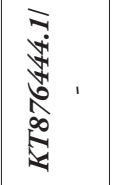 & 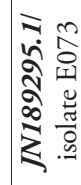 \\
\hline 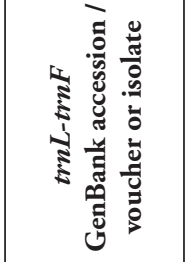 & 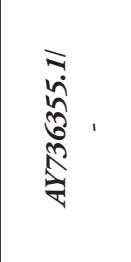 & 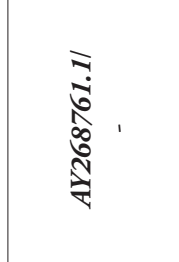 & 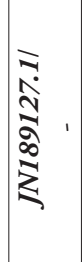 & 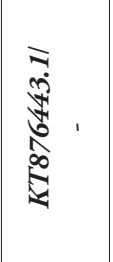 & 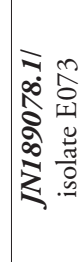 \\
\hline 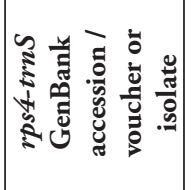 & 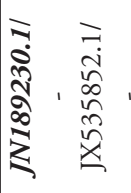 & $\begin{array}{l}\bar{\gamma} \\
\infty \\
\infty \\
\bar{\alpha} \\
\bar{\sigma}\end{array}$ & 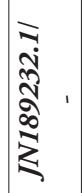 & 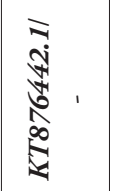 & $\begin{array}{ll} & 0 \\
\infty & 0 \\
0 & 0 \\
0 & 0 \\
0 & 0 \\
0 & 0 \\
\vdots & 0 \\
\vdots & 0\end{array}$ \\
\hline 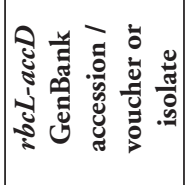 & 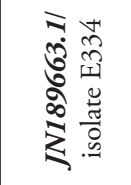 & 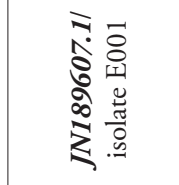 & 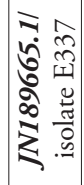 & 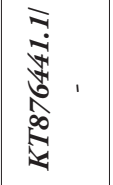 & 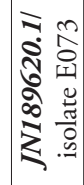 \\
\hline 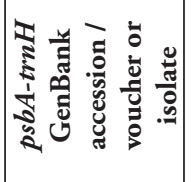 & 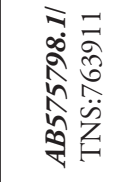 & $\begin{array}{l}\bar{\alpha} \\
\infty \\
\infty \\
\infty \\
\infty \\
\infty \\
\vdots\end{array}$ & 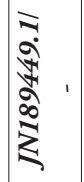 & 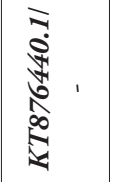 & 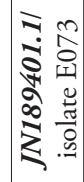 \\
\hline 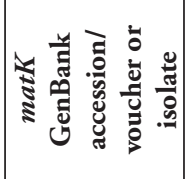 & & & & 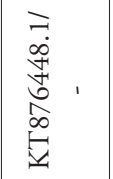 & 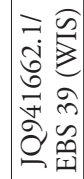 \\
\hline $\begin{array}{l}\text {. } \\
\text { कूँ } \\
\text { के }\end{array}$ & 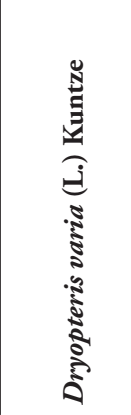 & 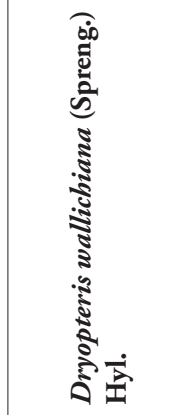 & 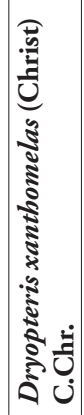 & 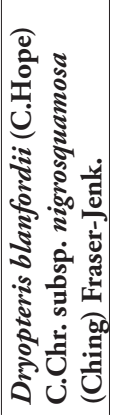 & 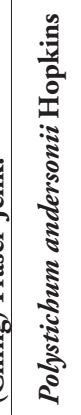 \\
\hline
\end{tabular}

